\title{
Luminescence spectra and kinetics of disordered solid solutions
}

Klochikhin, A.; Reznitsky, A.; Permogorov, S.; Breitkopf, T.; Grün, M.; Hetterich, M.; Klingshirn, C.; Lyssenko, Vadim; Langbein, Wolfgang Werner; Hvam, Jørn Märcher

Published in:

Physical Review B

Link to article, DOI:

10.1103/PhysRevB.59.12947

Publication date:

1999

Document Version

Publisher's PDF, also known as Version of record

Link back to DTU Orbit

Citation (APA):

Klochikhin, A., Reznitsky, A., Permogorov, S., Breitkopf, T., Grün, M., Hetterich, M., Klingshirn, C., Lyssenko, V., Langbein, W. W., \& Hvam, J. M. (1999). Luminescence spectra and kinetics of disordered solid solutions. Physical Review B, 59(20), 12947-12972. https://doi.org/10.1103/PhysRevB.59.12947

\section{General rights}

Copyright and moral rights for the publications made accessible in the public portal are retained by the authors and/or other copyright owners and it is a condition of accessing publications that users recognise and abide by the legal requirements associated with these rights.

- Users may download and print one copy of any publication from the public portal for the purpose of private study or research.

- You may not further distribute the material or use it for any profit-making activity or commercial gain

- You may freely distribute the URL identifying the publication in the public portal 


\title{
Luminescence spectra and kinetics of disordered solid solutions
}

\author{
A. Klochikhin \\ Petersburg Nuclear Physics Institute, St. Petersburg, Russia \\ A. Reznitsky and S. Permogorov \\ A. F. Ioffe Physical-Technical Institute, St. Petersburg, Russia
}

T. Breitkopf, M. Grün, M. Hetterich, and C. Klingshirn

Universität Karlsruhe, Institut für Angewandte Physik, 76128 Karlsruhe, Germany

V. Lyssenko

Institute of Microelectronics Technology, Chernogolovka, Moscow District, 142432, Russia

W. Langbein and J. M. Hvam

Mikroelectronik Centret, Technical University of Denmark, DK-2800, Lyngby, Denmark

(Received 22 June 1998; revised manuscript received 9 December 1998)

\begin{abstract}
We have studied both theoretically and experimentally the luminescence spectra and kinetics of crystalline, disordered solid solutions after pulsed excitation. First, we present the model calculations of the steady-state luminescence band shape caused by recombination of excitons localized in the wells of random potential induced by disorder. Classification of optically active tail states of the main exciton band into two groups is proposed. The majority of the states responsible for the optical absorption corresponds to the group of extended states belonging to the percolation cluster, whereas only a relatively small group of "radiative" states forms the steady-state luminescence band. The continuum percolation theory is applied to distinguish the "radiative", localized states, which are isolated in space and have no ways for nonradiative transitions along the tail states. It is found that the analysis of the exciton-phonon interaction gives the information about the character of the localization of excitons. We have shown that the model used describes quite well the experimental cw spectra of $\mathrm{CdS}_{(1-c)} \mathrm{Se}_{c}$ and $\mathrm{ZnSe}_{(1-c)} \mathrm{Te}_{c}$ solid solutions. Further, the experimental results are presented for the temporal evolution of the luminescence band. It is shown that the changes of band shape with time come from the interplay of population dynamics of extended states and spatially isolated "radiative" states. Finally, the measurements of the decay of the spectrally integrated luminescence intensity at long delay times are presented. It is shown that the observed temporal behavior can be described in terms of relaxation of separated pairs followed by subsequent exciton formation and radiative recombination. Electron tunneling processes are supposed to be responsible for the luminescence in the long-time limit at excitation below the exciton mobility edge. At excitation by photons with higher energies the diffusion of electrons can account for the observed behavior of the luminescence. [S0163-1829(99)11419-X]
\end{abstract}

\section{INTRODUCTION}

Wide application of disordered II-VI semiconductor solid solutions in modern quantum structures renews the interest to understand their optical properties.

In this paper, three interrelated aspects of luminescence of disordered solid solution are studied, namely, (i) the nature of the steady-state intrinsic luminescence band at low excitation, (ii) the effect of high-excitation density on the spectra of radiative recombination, and (iii) the long-time kinetics of the luminescence both at high and low density of excitation.

(i) The steady state intrinsic luminescence at a lowexcitation levels of many disordered systems ${ }^{1-13}$ including II-VI solid solutions, ${ }^{2-12}$ is supposed to arise from recombination of the excitons localized in the wells of a potential profile induced by the disorder. A common spectroscopic feature of such systems is a considerable redshift of the luminescence with respect to the maximum of exciton absorption [or with respect to the band-gap edge, as in the case of
$\alpha-\mathrm{Si}: \mathrm{H}$ (Ref. 1)], so that the fundamental emission band is situated in a spectral range where both the absorption coefficient and the density of fluctuation-induced states are small.

Street ${ }^{1}$ has supposed that the intrinsic luminescence band of $\alpha-\mathrm{Si}: \mathrm{H}$ can be understood with the assumption that among the tail states a special group of "radiative" localized states exists, which forms a relatively narrow band with the maximum at $1.6 \mathrm{eV}$. Cohen and Sturge $^{3}$ and Permogorov et $a .^{2}$ have claimed when studying II-VI solid solutions that the upper border of the luminescence band corresponds to the exciton mobility edge in the disordered system.

The results of the present paper are based on a theoretical approach, ${ }^{10-12}$ which incorporates both these ideas using as a background the model of solid solutions developed in Refs. 14 and 15. The calculations of the density of fluctuationinduced tail states are performed within the framework of a single band, two component model of solid solutions with diagonal disorder, which describes correctly the most common features of disorder in many solid solutions and is 
simple enough for numerical calculations of many characteristics of the tail states and the luminescence. It is supposed in the calculation of the composition dependence of tailing effects that two constituents are distributed randomly in a disordered sublattice. A significant deviation of the random distribution should lead to considerably different results.

The problem of tail-state localization is considered in an energy region $\omega>\omega_{0}$ where $\omega_{0}$ is defined by the condition $\mathcal{N}\left(\omega_{0}\right) a^{3}<1$. Here the localization energy $\omega$ is taken to be positive so that its value increases with the depth of the state, $a$ is a typical radius of the localized state, and $\mathcal{N}\left(\omega_{0}\right)$ is the number of tail states with localization energies exceeding $\omega_{0}$.

In the zero-th approximation, the tail states with energy exceeding $\omega_{0}$ are supposed to be localized. In solid solutions with anion substitution like $\mathrm{CdS}_{(1-c)}, \mathrm{Se}_{c}$, and $\mathrm{ZnSe}_{(1-c)} \mathrm{Te}_{c}$ localization is widely believed to be due to an effect of the random potential on the hole motion. Excitons are localized by clusters of attractive atoms at compositions below the threshold of the site percolation problem for the anion sublattice. At higher concentrations the localization is due to potential wells formed by a local excess concentration of the attractive component. In both cases the fluctuations responsible for the localization are considered as singly connected potential wells.

Further consideration of the tail-state character uses the approach of continuum percolation theory for overlapping spheres. ${ }^{16-22}$ In order to apply this theory a sphere with the radius $R_{\text {int }}$ is introduced around every potential well or cluster. The value of $R_{i n t}$, which is the parameter of the theory, should be chosen to provide proper spectral positions of luminescence and absorption maxima. The percolation theory allows to improve the zero-th approximation and to subdivide the tail states into the states formed by (1) spatially isolated clusters (or potential wells), by (2) complexes of clusters (or potential wells), which are referred further as superclusters, and (3) the states belonging to a percolation cluster.

The state with localization energy $\omega$ is treated as spatially isolated if no states with larger localization energy exist in the sphere of radius $R_{\text {int }}$ surrounding a given potential well. The state is considered as belonging to supercluster of size $n$, if there exists a path from the given state to any other of $(n-1)$ states with larger localization energy, which can be composed of steps that are smaller than $R_{\text {int }}$. At a certain localization energy $\omega_{M E}$ the supercluster of infinite size can be formed. This supercluster is regarded as a percolation cluster and $\omega_{M E}$ defines the mobility edge position.

On the basis of this classification the tail states can be divided into two groups with quite different radiative properties. The first group is formed by the states of isolated clusters (or potential wells) and by the ground states of superclusters of finite size. For these states only the radiative recombination is possible. We were able to calculate numerically the concentration and the energy distribution of these "radiative" states.

The second group constitutes the majority of the tail states and includes the excited states of superclusters and the states of percolation cluster. For these states nonradiative transitions into states with larger localization energy are possible.

Both types of states contribute to formation of the absorp- tion band of the exciton ground state, whereas the steadystate luminescence band at low intensity of excitation is supposed to be formed only by the "radiative" states of the fluctuation tail. The "radiative" states are situated mainly (but not exclusively) below the mobility edge and the luminescence band decreases sharply in the vicinity of the mobility edge. Even without the account for the exciton-phonon interaction the zero-phonon emission band is redshifted with respect to the zero-phonon absorption band for solid solution or the absorption edge for $\alpha-\mathrm{Si}: \mathrm{H}$. As it follows from the above model, the redshift of the luminescence band with respect to the absorption is an intrinsic property of disordered systems.

Phonon-assisted electronic transitions with simultaneous emission of optical and acoustical phonons cause the additional Stokes shift between the luminescence and absorption spectra. The account for the electron-phonon interaction allows us to describe quantitatively the shape of the luminescence spectra of different disordered systems in the limit of low excitation.

In particular, we were able to describe the experimental luminescence spectra of crystalline solid solutions $\mathrm{CdS}_{(1-c)} \mathrm{Se}_{c}$ (Refs. 10 and 11) and $\mathrm{ZnSe}_{(1-c)} \mathrm{Te}_{c} \cdot{ }^{12}$ The analysis of electron-phonon coupling shows that the strength of this interaction is considerably different for two models of localized exciton, namely, for excitons localized as a whole (model I) and for electrons bound by Coulomb interaction to holes localized by the fluctuating potential (model II). In the first model the electron can follow adiabatically the motion of the hole while it cannot do it in the second model. The comparison of calculated and experimental spectra shows that the localized excitons are formed by localized holes with electrons being coupled via the Coulomb interaction in $\mathrm{CdS}_{(1-c)} \mathrm{Se}_{c}$ at $c<0.4$ and in $\mathrm{ZnSe}_{(1-c)} \mathrm{Te}_{c}$ in the whole composition range studied.

(ii) The next subject of our studies is the temporal evolution of the luminescence band shape following excitation with a short light pulse. At short times after the excitation pulse the maximum of the time-resolved luminescence band is considerably shifted to higher energies with respect to its position observed in steady-state conditions. It can be supposed that the early stage of luminescence corresponds to the direct recombination of excitons created by photons. This stage covers the time interval of a few nanoseconds, which is comparable with the exciton radiative lifetime. Depending on the composition of solid solutions the luminescence decay during this initial stage of recombination shows a more or less pronounced deviation from a simple exponential behavior.

The high-energy shift of the luminescence maximum at short times can be attributed to the filling of the hole tail states including the extended states of the percolation cluster. Subsequent recombination and energy relaxation processes lead to the time-dependent changes of the band shape and its maximum position so that at sufficiently long times the luminescence spectrum regains the features of steady-state luminescence.

As it is known, the high-density excitation of semiconductor crystals leads to band-state filling and the screening of the Coulomb potential. ${ }^{23}$ As a consequence, the attractive electron-hole interaction decreases with increasing filling 
causing a reduction of the exciton binding energy. At the same time a renormalization of the single-particle energy results in a band-gap shrinkage. Both effects cancel each other because they have the same order of magnitude and opposite signs. In binary semiconductors the influence of electronhole plasma is mainly observed as a reduction of the exciton oscillator strength until the band energy reaches the exciton ground state at the Mott density when the exciton states disappear. ${ }^{23}$ The dynamics of band filling and of stimulated emission at high-excitation density have been investigated in $\mathrm{ZnSe}_{(1-c)} \mathrm{Te}_{c}$ and $\mathrm{CdS}_{(1-c)} \mathrm{Se}_{c}$ alloys in Refs. 24-29. The luminescence and gain spectroscopy of $\mathrm{CdS}_{(1-c)} \mathrm{Se}_{c}$ solid solutions under high-excitation density were reported in Ref. 30. It can be concluded, that the nonlinear electron-hole plasma effects are less pronounced in disordered systems than in the binary compounds. ${ }^{30}$ This finding can be considered as a consequence of the carrier localization by the fluctuating potential.

Other topics that have been already treated in these systems concern the coherent dynamics, ${ }^{28,31}$ the polarization memory for both linear and circular polarization, ${ }^{32-34}$ and the absolute luminescence yield of the localized states as function of temperature for various alloys. ${ }^{28}$

The studies of the $\mathrm{ZnSe}_{(1-c)} \mathrm{Te}_{c}$ and $\mathrm{CdS}_{(1-c)} \mathrm{Se}_{c}$ solid solutions presented in this paper were performed in a wide interval of the excitation power at which, nevertheless, the Coulomb potential was not screened though the considerable filling of the electronic and hole states was reached.

In agreement with the earlier data of Refs. $28,35-38$ we have found that more than $90 \%$ of the excitons recombine during the first few nanoseconds after the excitation pulse, while the remaining radiation lasts a few hundreds nanoseconds. Just after the high-power excitation pulse the initial luminescence band maximum is shifted toward higher energies. A comparison of the shift values in two different solid solutions $\mathrm{ZnSe}_{(1-c)} \mathrm{Te}_{c}$ and $\mathrm{CdS}_{(1-c)} \mathrm{Se}_{c}$ has shown an obvious correlation between the value of blueshift of the band maximum and the width of the fluctuation tail in absorption. The calculations, which we shall present, show that the form of the initial luminescence band can be described as a superposition of two overlapping bands, one of which is formed by "radiative" states and the other is due to states of percolation cluster. In the long-time limit only the band formed by radiative states survives.

(iii) Previous studies ${ }^{28,35-38}$ of the time kinetics of the luminescence at different spectral points have shown that the fast decay dominating the first stage of the process continuously transforms into the slow nonexponential behavior. Nevertheless, it was intuitively supposed that the spectrally integrated kinetics should follow an exponential decay with the radiative lifetime of excitons created by photons. The nonexponential behavior observed was considered as a consequence of population dynamics of excitons across the tail states. The experimental results of the present paper revealed a nonexponential behavior for the spectrally integrated luminescence kinetics even at very low-excitation densities, which definitely rules out the simple scheme of the exponential decay with the radiative lifetime of excitons. ${ }^{6,7}$ It means that the long-time kinetics cannot be described within the framework of the localized exciton-formation models, which were sufficient for simulations of the steady-state lumines- cence spectra. ${ }^{10-12}$ In this paper we shall try to consider possible reasons leading to the long-time survival of a small but clearly observable part of the excitations and generalize the model of localized exciton formation and recombination.

As a possible reason for the long-time nonexponential kinetics we are considering the creation of separated electronhole pairs in the process of energy relaxation of excitations generated by photons. It is supposed that both electrons and holes are subjected to the random potential of the disordered system. The time behavior of the luminescence intensity during the long-time nonexponential stage of relaxation depends on the energy of exciting photons and on the pulse power.

We assumed that even in the case of excitation below the mobility edge a part of absorbed photons produces "immobile" particles separated in space for which the radiative recombination is possible only through the tunneling, like in the case of donor-acceptor pairs ${ }^{39}$ and the obtained dependence presents a particular case of Becquerel's law. ${ }^{40}$ At excitation at and above the mobility edge the long-time luminescence kinetics acquires new features, which we describe using the results of the theory of diffusion controlled annihilation. ${ }^{41-43}$ In particular, the intermediate time kinetics shows stretched-exponential behavior with critical indices of "normal" $41-48$ or "anomalous", 41,43 diffusion. The longtime kinetics shows in this case a power law $t^{-3 / 2}$ for the decrease of intensity. This kind of dependence was obtained by Noolandi et al. ${ }^{50}$ in an approximation developed by Mozumder ${ }^{51}$ and it was observed in amorphous silicon. The other approach developed by Kuzovkov and Kotomin ${ }^{49}$ for the diffusion-controlled bimolecular reaction describes the inhomogeniety in the space distribution of the particles. As a result, power law follows in the long-time limit for the intensity decay $I(t) \sim t^{-3 / 2}$ in the case of equal concentrations of two kind of mobile and immobile particles.

The structure of the paper is as follows.

In Sec. II we present the main theoretical results.

In Sec. III the description of samples and other details of the experimental setup are given as well as the experimental data and their comparison with theory.

The last section of the paper is devoted to concluding remarks.

\section{THEORY}

Optical properties of a crystal can be described if eigenvalues and eigenfunction of the corresponding Hamiltonian are known. The complete solution of this problem remains still very difficult even for perfect crystals. Situation is even more complicated in disordered solids. Additional difficulties arise, on the one hand, from the absence of the long-range order and, on the other hand, from the composition dependence of the parameters of the system. An approximate approach to the problem has to take into account the peculiarities of the disordered systems.

An effective perturbation of the electronic states near the band edge occurs when an attractive center substitutes a host atom. For an isoelectronic substitution of a single atom in a three-dimensional regular crystal an important characteristic is the strength of perturbation of the electron states, which is characterized by the relation of the perturbation potential to its critical value $E_{c r}$, at which a localized state just appears. 
The strength of perturbation of each attractive atom decreases with an increase of the concentration of attractive centers because of the global shift of the averaged potential.

We will use the following classification of disordered systems in dependence of the strength of the isoelectronic perturbation and composition of the solution, which is convenient in the region of the tail states. The solid solution will be referred to as a strong scattering system if the perturbation is large enough to split off the bound state from the band edge when substituting a single atom or a small cluster of them. The lattice percolation theory can be applied for an accurate estimation of the number of tail states for strong-scattering systems in a relatively large region of compositions.

The weak-scattering limit will be related to the systems where the number of attractive atoms necessary to split off the localized state exceeds considerably unity.

An increase of the concentration of attractive centers lowers the averaged potential and results, thus, in a decrease of the number of localized states and a narrowing of the region of the tail states. As the consequence, a strong scattering system transforms into a weak scattering one. In general, there is a region of parameters where it is rather the question of convenience how to treat a given system.

The solid solution $\mathrm{ZnSe}_{1-c} \mathrm{Te}_{c}$ can be attributed at a low concentration of $\mathrm{Te}$ to the systems with strong scattering because already clusters consisting of two Te atoms create bound hole states above the valence-band edge. ${ }^{4}$ In this paper we are interested in the $\mathrm{ZnSe}_{1-c} \mathrm{Te}_{c}$ solid solution at concentrations $c$ of attractive Te atoms less than the critical value of the site percolation problem for the fcc sublattice, i.e., $c<p_{c} \approx 0.2$. According to the lattice percolation theory, ${ }^{16,17,52-54}$ randomly distributed Te atoms are able to form in the composition region $c<p_{c}$ only finite-size clusters.

The other systems under consideration are $\mathrm{CdS}_{(1-c)} \mathrm{Se}_{c}$ solid solutions in a wide composition interval. The $\mathrm{CdS}_{(1-c)} \mathrm{Se}_{c}$ solid solution presents a system where the perturbation by a single atom is weak and the bound states occur as a result of a large-scale fluctuation of composition formed by a considerable excess concentration of Se in some microscopic regions of the crystal.

Both systems show considerable decrease of tailing effects at high concentration of narrow-band component. ${ }^{4}$

\section{A. General characteristics of disorder effects in $\mathrm{ZnSe}_{1-c} \mathrm{Te}_{c}$ and $\mathrm{CdS}_{(1-c)} \mathrm{Se}_{c}$ solid solutions}

There are two characteristics of solid solutions, namely, the shift and the tailing of their band gap, which lead to a composition dependence of their optical properties.

A development of the theory of electron states in solid solutions ${ }^{55-65}$ is closely related with phenomenon of the nonlinear composition dependence or bowing of the band gap, which presents the deviation $\Delta E_{G}(c)$ from the simple linear interpolation of the band gap $E_{G}(c)$ as a function of composition

$$
E_{G}(c)=E_{G}(B)(1-c)+E_{G}(A) c-\Delta E_{G}(c) .
$$

Results of latest investigations in this field presented in Refs. 63-66 show that the perturbation of the electronic states due to the isoelectronic substitution has a complicated character.
According to Refs. 63-66, bowing is a result of selfconsistent changes of the electronic states due to the chemical perturbation and structural changes of the lattice due to the variations of chemical bond lengths.

The detailed analysis of the nonlinear part of the band gap variation with the composition in $\mathrm{ZnSe}_{1-c} \mathrm{Te}_{c}$ and $\mathrm{CdS}_{(1-c)} \mathrm{Se}_{c}$ solid solutions has shown ${ }^{4,5}$ that the bowing $\Delta E_{G}^{\exp }(c)$ as a function of the composition can be presented in the form of sum of two terms

$$
\Delta E_{G}^{e x p}(c)=\Delta E_{1}^{e x p}(c)+\Delta E_{2}^{e x p}(c),
$$

where $\Delta E_{1}^{e x p}(c)$ and $\Delta E_{2}^{e x p}(c)$ are different functions of the concentration. The larger term $\Delta E_{1}^{e x p}(c)$ is symmetrical in variables $c$ and $(1-c)$ and can be presented by the equation

$$
\Delta E_{1}^{e x p}(c)=b_{1} c(1-c) .
$$

Its maximum value for $\mathrm{ZnSe}_{1-c} \mathrm{Te}_{c}$ at $c=0.5$ amounts to about $0.25 \mathrm{eV}$. This value is comparable to the difference of the band gaps of $\mathrm{ZnSe}$ and $\mathrm{ZnTe}$ of 2.82 and $2.39 \mathrm{eV}$, ${ }^{67}$ respectively.

The maximum value of $\Delta E_{1}^{e x p}(c)$ for $\mathrm{CdS}_{(1-c)} \mathrm{Se}_{c}$ is equal to $\approx 0.055 \mathrm{eV}$ while the difference of the band gaps of $\mathrm{CdS}$ and CdSe crystals is equal to $0.72 \mathrm{eV}$.

The second term $\Delta E_{2}^{e x p}(c)$ reaches its maximum value at $c \approx 0.15-0.20$ and it is equal to $\approx 0.1 \mathrm{eV}$ for $\mathrm{ZnSe}_{1-c} \mathrm{Te}_{c}$ and to $\approx 0.030 \mathrm{eV}$ for $\mathrm{CdS}_{(1-c)} \mathrm{Se}_{c} .4,5$

Both absorption and luminescence spectra of $\mathrm{ZnSe}_{1-c} \mathrm{Te}_{c}$ and $\mathrm{CdS}_{(1-c)} \mathrm{Se}_{c}$ solid solutions show evidences of localization effects due to composition fluctuations. The form of the fundamental absorption edge follows the simple Urbach law characterized by the Urbach tailing parameter $\varepsilon_{U}(c)$, which defines the exponential decreasing absorption tail. The maximum values of $\varepsilon_{U}(c)$ in $\mathrm{ZnSe}_{1-c} \mathrm{Te}_{c}$ and $\mathrm{CdS}_{(1-c)} \mathrm{Se}_{c}$ solid solutions are equal to $\varepsilon_{U}(c) \approx 0.030$ and $\approx 0.005 \mathrm{eV}$, respectively. The composition dependences of $\varepsilon_{U}(c)$ coincide in both crystals practically with those of $\Delta E_{2}^{\exp }(c)$, which allows one to treat the term $\Delta E_{2}(c)$ as a result of the same composition fluctuations that are responsible for the tailing. Both these functions can be approximated by the equation ${ }^{14}$

$$
\Delta E_{2}^{e x p}(c), \varepsilon_{U}(c) \sim \frac{(1-c)^{5 / 2}}{\ln (1 / c)} .
$$

\section{B. Density of fluctuation states in systems with diagonal disorder}

The influence of disorder on the optical properties depends strongly on the number of states split off the band edge. The most direct optical characteristics concerned with this number of states is the broadening of the exciton ground-state transition, though the exciton-phonon interaction has the tendency to hide or to increase the effect. Substituting atoms form a great variety of configurations and the general problem of disordered systems is how to find and to enumerate those responsible for the tailing. The energy interval of interest is rather large enough and both size and number of clusters responsible for the localization increase enormously with the growth of the localization energy and quickly reach the values inaccessible for a direct calculation 
even for strongly scattering systems at concentrations below the sublattice percolation threshold $c<p_{c}$.

Thus, this fact requires an approach that allows one to separate the problem of estimation of the number of localized states from a calculation of the energy dependence of the density of states and to solve these problems by means of quite different approximations.

(1) Within a narrow energy interval the number of states below the band edge is estimated using (a) mathematically accurate equations of lattice percolation theory for the case $c<p_{c}$ in the strong scattering limit, or (b) the simple model of potential wells at compositions $c<\left(1-p_{c}\right)$, which gives the correct order of the magnitude of the density of states (DOS). If it is necessary the obtained DOS can be fitted to the experimental data on the exciton band broadening.

(2) Calculation of the energy dependence of DOS in effective-mass approximation in both cases (a) and (b) with the help of a variational procedure, which presents in its essence an interpolation between two limits, namely, between the region of the Lifshitz border where the procedure gives an adequate result and the region near the band edge where the density of states is already a function only weakly dependent on energy. The calculated DOS should be normalized properly by the results of the previous point (1).

\section{Model Hamiltonian}

We suppose that electron-hole pairs, which take part in absorption and luminescence in the tail region of the exciton ground state in the solid solution under consideration, are localized in potential wells due to disorder effect on the states of the valence band while electrons are bound to holes by Coulomb interaction. We have two limiting possibilities in this case. If the electron can follow the hole motion adiabatically we have to consider the exciton localized as the whole (model I). The other situation can be realized if the hole motion is too fast and the electron interacts with averaged distribution of the hole density (model II).

Inasmuch as the electron mass is considerably smaller than that of the hole we neglect the difference in masses of the exciton and hole, and will describe the localized states in both cases by the same single-particle Hamiltonian. We neglect also the small difference in the electron-hole Coulomb interaction for these two models. We will take into account only the ground state of the electron-hole Coulomb problem, which will be included into the considerations of the spectral density of the tail states (subsection F) and in calculations of the exciton phonon interaction (subsection J). A significant difference of these two models becomes obvious in the consideration of the exciton-phonon interaction (subsection $\mathrm{J}$ ).

Let a macroscopic volume $V$ of the crystal containing $N$ lattice sites be randomly filled with two sorts of atoms $A$ and $B$. The average number of atoms $A$ is equal to $N_{A}=c N$ and that of atoms $B$ is $N_{B}=(1-c) N$. Atoms $A$ are supposed to be attractive centers and $c$ is their concentration. The singleband Hamiltonian of the system is written as

$$
H=-\sum_{\mathbf{n}, \mathbf{m}} \Psi_{\mathbf{n}} W_{\mathbf{m}}\left(\Psi_{\mathbf{n}+\mathbf{m}}-\Psi_{\mathbf{n}}\right)+\sum_{\mathbf{n}} E_{\mathbf{n}} \Psi_{\mathbf{n}}^{2}
$$

The wave functions $\Psi_{\mathbf{n}}$ can be taken in many cases to be real. The diagonal matrix element $E_{\mathbf{n}}$ is equal to $E_{A}$ if the site is occupied with atom $A$ and to $E_{B}$ in the opposite case.

In the limiting cases $c=0$ or $c=1 \mathrm{Eq}$. (4) presents the Hamiltonian of the regular crystal $B$ or $A$ with all $E_{\mathbf{n}}$ being either equal to $E_{B}$ or $E_{A}$. Using the plane-wave representation we have in these cases

$$
H_{\mathbf{q}}^{\alpha}=\varepsilon_{\mathbf{q}}+E_{\alpha},
$$

where $\alpha$ is $A$ or $B$ and the relation

$$
\varepsilon_{\mathbf{q}}=W_{0}-W_{\mathbf{q}}
$$

describes the electronic band dispersion. The off-diagonal matrix element $W_{\mathbf{m}}$ defining the band dispersion is supposed to be independent on the composition. We suppose in the calculations $E_{B}>E_{A}$.

\section{The single-particle Green's function and the density of states}

The Green's function $G_{\mathbf{n m}}^{\alpha}(\omega)$ of the pure crystal $A$ or $B$ can be written as

$$
G_{\mathbf{n m}}^{\alpha}(\omega)=\left\{\omega \mathbf{I}-\mathbf{H}^{\alpha}\right\}_{\mathbf{n m}}^{-1}=\frac{1}{N} \sum_{\mathbf{q}} \frac{e^{i \mathbf{q}\left(R_{\mathbf{n}}-R_{\mathbf{m}}\right)}}{\omega-\varepsilon_{\mathbf{q}}-E_{\alpha}},
$$

where $\mathbf{R}_{\mathbf{n}}$ is a radius-vector of the lattice site $\mathbf{n}, \varepsilon_{\mathbf{q}}^{\alpha}$ is the self-energy of an electron in the band with wave vector $\mathbf{q}$, and I is the unit matrix. Equation (7) defines the Green's function uniquely at energies $\omega$ outside the band, where this function is real. Inside the band, we can define retardant and advanced Green's functions by the introduction of a small imaginary energy parameter in the denominator of the Green's function $\omega \rightarrow \omega \pm i 0$.

For a random distribution of the atoms $A$ and $B$ the Green's function is dependent on the composition and atoms positions

$$
G_{\mathbf{n m}}(\omega)=\left\{\omega \mathbf{I}+\mathbf{H}^{v c}-\Delta\right\}_{\mathbf{n m}}^{-1} .
$$

The matrixes $\mathbf{H}^{v c}$ and $\boldsymbol{\Delta}$ are defined by the equations

$$
\mathbf{H}_{\mathbf{n m}}^{v c}=W_{\mathbf{n}-\mathbf{m}}-W_{0} \delta_{\mathbf{n m}} ; \Delta=\Delta_{\mathbf{n}} \delta_{\mathbf{n m}} .
$$

Here,

$$
E_{G}(c)=c E_{A}+(1-c) E_{B}
$$

is the linear in concentration shift, which results from averaged value of $E_{\mathbf{n}}$ in a lattice site. Let $E_{G}$ defind by Eq. (10) be a frame of reference for energy and $\omega>0$ in the region of localized states. Then diagonal matrix elements are compositionally dependent functions

$$
\Delta_{\mathbf{n}}=E_{\mathbf{n}}-E_{G}(c)
$$

The eigenvalues and eigenfunctions of the Hamiltonian at any realization of a random distribution of two kind atoms can be found by means of diagonalizing the $N$-rank matrix, which is formed by columns like

$$
\omega_{\Lambda} \varphi_{\Lambda}^{\Delta}(\mathbf{n})+\sum_{m}\left(H_{\mathbf{n m}}^{v c}-\Delta_{\mathbf{n}} \delta_{\mathbf{n m}}\right) \varphi_{\Lambda}^{\Delta}(\mathbf{m})=0
$$

The density of states of disordered systems can be found as the result of the averaging procedure, i.e., summing over the DOS corresponding to all possible realizations of the disor- 
der with the weight multipliers $P_{\Delta}$, which are equal to the probability that a given variant of random distribution occurs. Taking into account all of the possible quantum numbers $\Lambda$ we obtain

$$
\begin{aligned}
\rho(\omega)= & \frac{1}{2 \pi} \int_{-\infty}^{\infty} \sum_{\Delta} P_{\Delta} \sum_{\Lambda} d \tau_{\Lambda} \sum_{\mathbf{n}}\left|\varphi_{\Lambda}^{\Delta}(\mathbf{n})\right|^{2} \\
& \times \exp \left[-i\left(\omega \mathbf{I}+\mathbf{H}^{v c}-\boldsymbol{\Delta}\right)_{\Lambda}{ }_{\Lambda} \tau_{\Lambda}\right]
\end{aligned}
$$

where

$$
\begin{aligned}
{[\omega \mathbf{I}} & \left.+\mathbf{H}^{\mathbf{v}}-\Delta\right]_{\Lambda \Lambda} \\
& =\sum_{\mathbf{n}, \mathbf{m}} \varphi_{\Lambda}^{\Delta}(\mathbf{n})\left(\omega \delta_{\mathbf{n m}}+H_{\mathbf{n m}}^{v c}-\Delta_{\mathbf{n}} \delta_{\mathbf{n m}}\right) \varphi_{\Lambda}^{\Delta}(\mathbf{m}) .
\end{aligned}
$$

Performing the integration over $\tau_{\Lambda}$ we transform $\rho(\omega)$ into the form

$$
\rho(\omega)=\sum_{\Delta} P_{\Delta} \sum_{\Lambda} \sum_{\mathbf{n}}\left|\varphi_{\Lambda}^{\Delta}(\mathbf{n})\right|^{2} \delta\left(\omega-\omega_{\Lambda}^{\Delta}\right) .
$$

Here we have denoted eigenvalues of the Hamiltonian at a given realization of disorder as $\omega_{\Lambda}^{\Delta}$. Eigenfunctions $\varphi_{\Lambda}^{\Delta}$ for the localized states can be normalized to unity.

In order to compare the calculated functions of the localization energy with experimental data we need a frame of reference for energy variables.

There is a problem concerning the large value of the total band gap bowing $\Delta E_{G}(c)^{\exp }$ as compared with the localization energies of the tail states. The frame of reference obtained by means of a simple linear interpolation of the band gaps like Eq. (10) would lead to a considerable overestimation of the localization energies of the tail states. Taking into account that there are theoretical arguments ${ }^{55-66}$ and experimental findings that allow us to conclude that $\Delta E_{1}^{\exp }(c)$ does not influence the localization, we will use in our further consideration a subtraction of the symmetrical part of the band gap bowing, which depends on the composition of the solid solution as it is given by Eq. (2).

After subtraction $\Delta E_{1}^{\exp }(c)$ the frame of reference for the experimental energies is given by the equation

$$
E_{G}^{e x p}(c)=c E_{G}^{A}+(1-c) E_{G}^{B}-\Delta E_{1}^{e x p}(c) .
$$

The value of the $\Delta E_{1}^{e x p}(c)$ itself can be defined accurately from the experimental data if the bowing is known in the region of large concentrations of the narrow-band component where the second part of the bowing $\Delta E_{2}^{\exp }(c)$ is of no importance. 5

The function corresponding in our calculations to $\Delta E_{1}^{\exp }(c)$ is the band-edge bowing due to single-site fluctuations $\Delta E_{1}(c)$. There are different approximations given in Refs. 55-66 which were used to calculate the band-edge bowing. In the considered model the scattering from the single-site fluctuations presents the only mechanism that produces the symmetrical part of the band-edge bowing. In second-order perturbation theory

$$
\Delta E_{1}(c)=\Delta^{2} G_{\mathbf{n n}}^{v c}(0) c(1-c),
$$

where

$$
G_{\mathbf{n m}}^{v c}(\omega)=\left(\omega \mathbf{I}-\mathbf{H}^{v c}\right)_{\mathbf{n m}}^{-1}
$$

is the Green's function in virtual crystal approximation, and

$$
\Delta=\left|E_{B}-E_{A}\right|
$$

is the amplitude of the fluctuation potential. Taking into account that

$$
\left[G_{\mathbf{n n}}^{v c}(0)\right]^{-1} \equiv E_{c r}
$$

is equal to the single-site critical value of the perturbation potential we see that $\Delta E_{1}(c)$ depends on the same parameters of the system as the Urbach energy.

In spite of the fact that Eq. (17) does not provide an adequate description of band-edge bowing in the general case (discussion of this problem can be found in Refs. 6062) we may expect that in the simple model under consideration the calculated value of the bowing has the correct order of magnitude if the parameters of the model Hamiltonian $\Delta$ and $\Delta / E_{c r}$ are found as a result of a fitting procedure of the form of the absorption spectra.

The experimental value of the symmetrical part of the band-gap bowing $\Delta E_{1}^{\text {exp }}(c)$ and the calculated band edge bowing $\Delta E_{1}(c)$ obey for both $\mathrm{CdS}_{(1-c)} \mathrm{Se}_{c}$ and $\mathrm{ZnSe}_{(1-c)} \mathrm{Te}_{c}$ the inequality

$$
\Delta E_{1}(c) \leqslant \Delta E_{1}^{\exp }(c) .
$$

This fact shows that contributions from the valence bands define mainly these values.

The obtained band-edge bowing of Eq. (17) gives a constant shift of the frame of reference of Eq. (10) for all energy variables in the theoretical calculation excluding this value from the calculations of the localization energies.

\section{Strong-scattering limit, $c<p_{c}$. Isolated cluster approximation, sum rule}

The limit of small concentration $c \ll 1$ of strongly attractive centers is closely related with the strong localperturbation problem solved in Ref. 68 for electrons and in Ref. 69 for phonons (see also Refs. 70 and 71, and references therein). It corresponds to the case where the depth of the potential wells of the lattice sites occupied by atoms $A$ is large as compared with its critical magnitude, i.e.,

$$
(1-c) \Delta \gg\left|G_{\mathbf{n n}}^{v c}(0)\right|^{-1} .
$$

If the interatomic distances between atoms $A$ exceed the radius of the bound state appearing at each lattice site occupied by atoms $A$ then Eq. (12) reduces in the region of the bound states with accuracy up to terms of the order of $c^{2}$ to the Koster-Slater-Lifshitz equation (see Refs. 68-71)

$$
\left[1+\Delta G_{\mathbf{n n}}^{v c}(\omega)\right] \varphi_{\Lambda}^{\Delta}(\mathbf{n})=0 .
$$

At small but finite concentrations of the attractive centers there exists a finite probability to find pairs and larger clusters of attractive centers. ${ }^{72}$

We develop here a straightforward approach to the problem in the strong scattering limit at $c<p_{c}$ when atoms $A$ form only clusters of finite size. Let us assume in the first 
approximation that the cluster wave functions of the localized states are not overlapping and treat the medium surrounding a cluster in the virtual crystal approximation. Later, we will take into account composition fluctuations outside the cluster.

For any cluster, the equation of motion can be presented in this approximation in the form

$$
\left[\mathbf{I}+\mathbf{g}^{v c}(\omega) \Delta\right]_{\mathrm{nm}} \varphi_{\Lambda}^{\Delta}(\mathbf{m})=0,
$$

where $\mathbf{n}$ and $\mathbf{m}$ take the values corresponding the lattice sites coordinates occupied by the cluster and $\mathbf{g}^{v c}(\omega)$ is the fragment of the Green's-function matrix restricted by the region of the cluster. Using an approach developed in the theory of lattice dynamics, ${ }^{73}$ we find the eigenfunctions and eigenvalues of the matrix

$$
\left[\mathbf{g}^{v c}(\omega) \boldsymbol{\Delta}\right]
$$

the rank of which is equal to the size of the cluster considered

$$
\sum_{\mathbf{m} \in \mathcal{C}_{s t, \kappa}}\left[\mathbf{g}^{v c}(\omega) \Delta\right]_{\mathbf{n}, \mathbf{m}} \Phi^{\sigma}(\mathbf{m}, \omega)=\lambda^{\sigma}(\omega) \Phi^{\sigma}(\mathbf{n}, \omega)
$$

where summing is performed over the region occupied by the cluster, which contains $s$ attractive centers and $t$ perimeter sites. Index $\kappa$ enumerates different space configurations of the cluster. The eigenfunctions form the complete orthonormal set of vectors obeying the equation ${ }^{74}$

$$
\sum_{\sigma} \Phi^{\sigma}(\mathbf{m}, \omega) \Phi^{\sigma}(\mathbf{n}, \omega)=\delta_{\mathbf{n m}}
$$

With the help of these eigenfunctions and eigenvalues the matrix $\left[\mathbf{I}+\mathbf{g}^{v c}(\omega) \boldsymbol{\Delta}\right]^{-1}$ can be presented as

$$
\left[\mathbf{I}+\mathbf{g}^{v c}(\omega) \boldsymbol{\Delta}\right]_{\mathbf{n m}}^{-1}=\sum_{\sigma} \frac{\Phi^{\sigma}(\mathbf{n}, \omega) \Phi^{\sigma}(\mathbf{m}, \omega)}{1-\lambda_{\sigma}(\omega)} .
$$

The localized state will be split off if at least for one of the $\sigma$ there exists the value $\omega=\omega_{l o c}>0$, for which the equality is fulfilled

$$
\lambda_{\sigma}\left(\omega_{l o c}\right)=1 .
$$

The general rule is that, the nodeless state splits off first and this state has the deepest localization energy and is most important for the optical properties of a system. The wave function of the localized states can be presented as

$$
\begin{aligned}
\phi^{\sigma}(\mathbf{n})= & \sum_{\mathbf{m} \in \mathcal{C}_{s t, \kappa}} G_{\mathbf{n}, \mathbf{m}}^{v c}(\omega) \Phi_{\mathbf{m}}^{\sigma}(\omega) \\
& \times\left|\sum_{\mathbf{n}, \mathbf{m} \in \mathcal{C}_{s t, \kappa}} \Phi_{\mathbf{n}}^{\sigma} G_{\mathbf{n}, \mathbf{m}}^{\prime v c}(\omega) \Phi_{\mathbf{m}}^{\sigma}\right|^{-1 / 2},
\end{aligned}
$$

where

$$
G_{\mathbf{n}, \mathbf{m}}^{\prime v c}(\omega)=\frac{\partial}{\partial \omega} G_{\mathbf{n}, \mathbf{m}}^{v c}(\omega)
$$

Let us write the probability of realizing a cluster containing $s$ atoms $A$ and having a perimeter of $t$ atoms $B$ as

$$
g_{s t} c^{s}(1-c)^{t}
$$

where $g_{s t}$ is the number per lattice site of different space configurations of clusters, which have a coinciding number of atoms $\mathrm{A}$ and $\mathrm{B}$. Taking into account the nodeless bound states only we can write for the density of states

$$
\rho(\omega)=\sum_{s} \sum_{\kappa=1}^{g_{s t}} \sum_{\mathbf{n}}\left|\phi_{\mathbf{n}}^{s t, \kappa}\right|^{2} c^{s}(1-c)^{t} \delta\left(\omega-\omega_{l o c}^{s t, \kappa}\right) .
$$

The integrated DOS per lattice site can be written

$$
\mathcal{N}(0)=\int_{0}^{E_{L}} \rho(\omega) d \omega=\sum_{s t, \kappa} g_{s t} c^{s}(1-c)^{t}=\sum_{s} n_{s}(c),
$$

where $E_{L}$ is the Lifshitz border for the solid solution. The right-hand side of the equation coincides with the total number of clusters per lattice site.

Sums like Eq. (32) are to appropriate accuracy determined by their lower limit, i.e., by their first few terms at any concentration. As soon as a few first values of $n_{s}(c)$ are known for the different lattices, sums like this one can be practically used to estimate the number of states splitting off the band edge in the case of the strong scattering limit.

According to Refs. 52 and 53 the numbers $n_{s}(c)$ are equal to

$$
\begin{gathered}
n_{1}(c)=c(1-c)^{12} ; n_{2}(c)=12 c^{2}(1-c)^{18} ; \\
n_{3}(c)=c^{3}\left[24(1-c)^{23}+126(1-c)^{24}\right]
\end{gathered}
$$

for fcc lattice. The quantity $n_{4}(c)$ can be estimated using Eq. (33) as

$$
n_{4}(c) \approx 10^{3} c^{4}(1-c)^{30} .
$$

Further useful information can be obtained if the dependence of the localization energy on the number of atoms $A$ in a cluster is known at least approximately. This allows one to estimate the energy dependence of the integrated DOS as well as the DOS itself.

The maximum number of the localized states that can split off the cluster containing $s$ attractive centers is equal to $s$. The mean numbers of clusters $n_{s}(c)$ of size $s$ are normalized according to equation

$$
N_{A}=N \sum_{s=1}^{\infty} n_{s}(c) s
$$

where $N$ is the number of lattice sites and $N_{A}$ is the mean number of atoms A per unit volume. This equation defines the upper limit of the number of localized states. This estimation can be useful in the limit of very strong scattering systems.

\section{Effect of fluctuations on localized cluster states. Perturbation theory approach}

In the previous consideration we supposed that the medium surrounding the clusters can be approximated by the virtual crystal. In order to estimate the role of composition fluctuations outside the cluster we will substitute the ob- 
tained solution into Eq. (13) and find the first correction to the DOS due to fluctuations. As a result we have

$$
\begin{aligned}
\rho(\omega)= & \frac{1}{2 \pi} \int_{-\infty}^{\infty} d \tau \sum_{s} \sum_{\kappa=1}^{g_{s t}} \sum_{\mathbf{n}}\left|\phi_{\mathbf{n}}^{s t, \kappa}\right|^{2} c^{s}(1-c)^{t} \\
& \times \exp \left[-i\left(\omega-\omega_{l o c}^{s t, \kappa}\right) \tau\right] \\
& \times\left(\operatorname { e x p } \left(-i \sum_{\left.\left.\mathbf{n} \ni \mathcal{C}_{s t, \kappa} \Delta_{\mathbf{n}}\left|\phi_{\mathbf{n}}^{s t, \kappa}\right|^{2} \tau\right)\right),}\right.\right.
\end{aligned}
$$

where $\langle\cdots\rangle$ means averaging over all possible realizations of lattice site filling except a given cluster. At a random distribution each of the lattice sites is occupied by atom $A$ with probability $c$ and with probability $(1-c)$ by atom $B$. Taking into account that the averaging exponent is splitting into the product of exponents and that each of the multipliers is averaging independently we obtain

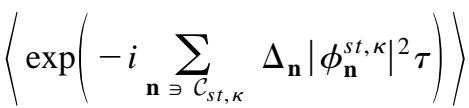

$$
\begin{aligned}
& =\prod_{\mathbf{n} \ni}^{N}\left[c \exp \left(-i \Delta_{A}\left|\phi_{\mathbf{n}}^{s t, \kappa}\right|^{2} \tau\right)\right. \\
& \left.+(1-c) \exp \left(-i \Delta_{B}\left|\phi_{\mathbf{n}}^{s t, \kappa}\right|^{2} \tau\right)\right] .
\end{aligned}
$$

Here

$$
\begin{gathered}
\Delta_{A}=E_{A}-E_{G}=-(1-c) \Delta, \\
\Delta_{B}=E_{B}-E_{G}=c \Delta .
\end{gathered}
$$

The averaged expression for the DOS can then be written as

$$
\begin{aligned}
\rho(\omega)= & \frac{1}{2 \pi} \int_{-\infty}^{\infty} d \tau \sum_{s} \sum_{\kappa=1}^{g_{s t}} \sum_{\mathbf{n}}\left|\phi_{\mathbf{n}}^{s t, \kappa}\right|^{2} c^{s}(1-c)^{t} \\
& \times \exp \left\{-i\left(\omega-\omega_{l o c}^{s t, \kappa}\right) \tau+\sum_{\mathbf{n} \ni \mathcal{C}_{s t, \kappa}} \ln \left[R_{\mathbf{n}}(\tau)\right]\right\},
\end{aligned}
$$

where

$$
\begin{aligned}
R_{\mathbf{n}}(\tau)= & c \exp \left[i \Delta(1-c)\left|\phi_{\mathbf{n}}^{s t, \kappa}\right|^{2} \tau\right] \\
& +(1-c) \exp \left(-i \Delta c\left|\phi_{\mathbf{n}}^{s t, \kappa}\right|^{2} \tau\right) .
\end{aligned}
$$

Restricting to the first nonzero cumulant we have for the DOS

$$
\begin{aligned}
\rho(\omega)= & \frac{1}{2 \pi} \int_{-\infty}^{\infty} d \tau \sum_{s} \sum_{\kappa=1}^{g_{s t}} \sum_{\mathbf{n}}\left|\phi_{\mathbf{n}}^{s t, \kappa}\right|^{2} c^{s}(1-c)^{t} \\
& \times \exp \left[-i\left(\omega-\omega_{l o c}^{s t, \kappa}\right) \tau-\gamma_{s t, \kappa}^{2} \tau^{2} / 2\right]
\end{aligned}
$$

where

$$
\gamma_{s t, \kappa}^{2}=\sum_{\mathbf{n} \ni \mathcal{C}_{s t, \kappa}} c(1-c) \Delta^{2}\left[\left|\phi_{\mathbf{n}}^{s t, \kappa}\right|^{2}\right]^{2} .
$$

The summing in the last expression has to be performed over the lattice sites outside the given cluster. After calculating the integral over $\tau$ we have

$$
\begin{aligned}
\rho(\omega)= & \sum_{s} \sum_{\kappa=1}^{g_{s t}} \sum_{\mathbf{n}}\left|\phi_{\mathbf{n}}^{s t, \kappa}\right|^{2} c^{s}(1-c)^{t} \\
& \times \frac{1}{\sqrt{2 \pi \gamma_{s t, \kappa}^{2}}} \exp \left[-\left(\omega-\omega_{l o c}^{s t, \kappa}\right)^{2} /\left(2 \gamma_{s t, \kappa}^{2}\right)\right]
\end{aligned}
$$

The expression obtained differs from Eq. (31) because the localization energy in the last equation is defined with accuracy $\gamma_{s t, \kappa}$. If the localization energy and $\gamma_{s t, \kappa}$ are comparable then the number of states split off the band edge decreases due to fluctuations as compared to the case without fluctuations. This means that

$$
\mathcal{N}(0)=\int_{0}^{E_{L}} \rho(\omega) d \omega \leqslant \sum_{s} n_{s}(c) .
$$

The result obtained is restricted to the region where the perturbation theory is applicable, i.e., it is the better the lower the cluster concentration and the larger the localization energy are.

\section{Effect of fluctuations on localized cluster states. Variational procedure}

To extend the region of the cluster approach we apply the variational procedure to consider fluctuations of the surrounding medium. For further calculations it is convenient to rewrite the Fourier integral of Eq. (36) as a Laplace transformation

$$
\begin{aligned}
\rho(\omega)= & \frac{1}{2 \pi} \int_{-\infty}^{\infty} d \tau \sum_{s} \sum_{\kappa=1}^{g_{s t}} \sum_{\mathbf{n}}\left|\phi_{\mathbf{n}}^{s t, \kappa}\right|^{2} c^{s}(1-c)^{t} \\
& \times \exp \left\{-i\left[\sum_{\mathbf{n m}} \phi_{\mathbf{n}}^{s t, \kappa}\left(H_{\mathbf{n m}}^{v c}+\omega \delta_{\mathbf{n m}}\right) \phi_{\mathbf{m}}^{s t, \kappa}\right]\left(\tau-i \theta_{s t, \kappa}\right)\right. \\
& +\sum_{\left.\mathbf{n} \ni \mathcal{C}_{s t, \kappa} \ln \left[R_{\mathbf{n}}\left(\tau-i \theta_{s t, \kappa}\right)\right]\right\}}
\end{aligned}
$$

Here, $\phi_{\mathbf{m}}^{s t, \kappa}$ is the trial wave function of the localized state for the given cluster, and $\theta_{s t, \kappa}$ is the corresponding parameter of the Laplace transformation. An important detail of the problem is concerned with the great variety of the cluster configurations. As a result the trial functions for the variational procedure should be chosen for each of the clusters individually. Neglecting the possible overlapping of the wave functions of clusters we consider in this case isolated clusters. The problem consists in the solution of the equations, which have the form

$$
\sum_{\mathbf{n m}}\left[H_{\mathbf{n m}}^{v c}+\omega \delta_{\mathbf{n m}}\right] \phi_{\mathbf{m}}^{s t, \kappa}+U_{s t, \kappa}(\mathbf{n}) \phi_{\mathbf{n}}^{s t, \kappa}=0 .
$$

The potential energy inside the cluster is defined uniquely by the configuration of the cluster. Outside the cluster, the 
most probable potential energy has to be found. As a result of this optimization we have for the potential energy $U_{s t, \kappa}(\mathbf{n})$ for cluster $s t, \kappa$

$$
U_{s t, \kappa}(\mathbf{n})=\left\{\begin{array}{cc}
\Delta_{\mathbf{n}} & \mathbf{n} \in \mathcal{C}_{s t, \kappa} \\
-\frac{\partial}{\partial \theta_{s t, \kappa}\left|\phi_{\mathbf{n}}^{s t, \kappa}\right|^{2}} \ln R_{\mathbf{n}}\left(-i \theta_{s t, \kappa}\right) & \mathbf{n} \ni \mathcal{C}_{s t, \kappa},
\end{array}\right.
$$

where

$$
\begin{aligned}
& -\frac{\partial \ln R_{\mathbf{n}}\left(-i \theta_{s t, \kappa}\right)}{\partial \theta_{s t, \kappa}\left|\phi_{\mathbf{n}}^{s t, \kappa}\right|^{2}} \\
& \quad=-(1-c) \Delta\left[1-\frac{\exp \left(-\Delta \theta_{s t, \kappa}\left|\phi_{\mathbf{n}}^{s t, \kappa}\right|^{2}\right)}{c+(1-c) \exp \left(-\Delta \theta_{s t, \kappa}\left|\phi_{\mathbf{n}}^{s t, \kappa}\right|^{2}\right)}\right] .
\end{aligned}
$$

The self-consistent solutions of the equations of "motion" give functions $\phi_{\mathbf{n}}^{s t, \kappa}$ and define parameters of the Laplace transformation $\theta_{s t, \kappa}$.

Presenting in Eq. (44) $\ln \left[R_{\mathbf{n}}\left(\tau-i \theta_{s t, \kappa}\right)\right]$ in form of a Taylor series in $\tau$ and keeping terms up to $\tau^{2}$ we transform the integral of Eq. (44) to a Gaussian. Integration leads to a density of states, which can be rewritten using the equation of motion (45) as

$$
\begin{aligned}
\rho(\omega)= & \sum_{s} \sum_{\kappa=1}^{g_{s t}} \sum_{\mathbf{n}}\left|\phi_{\mathbf{n}}^{s t, \kappa}\right|^{2} c^{s}(1-c)^{t} \\
& \times \frac{1}{\sqrt{2 \pi \gamma_{s t, \kappa}^{2}(\omega)}} \exp \left\{\sum _ { \mathbf { n } \ni \mathcal { C } _ { s t , \kappa } } \left[\ln \left(\frac{c}{c+p(\mathbf{n})}\right)^{c+p(\mathbf{n})}\right.\right. \\
& \left.\left.\times\left(\frac{1-c}{1-c-p(\mathbf{n})}\right)^{1-c-p(\mathbf{n})}\right]\right\}
\end{aligned}
$$

where $p(\mathbf{n})=-U_{s t, \kappa}(\mathbf{n}) / \Delta$, and

$$
\begin{gathered}
\gamma_{s t, \kappa}^{2}(\omega)=\frac{m_{2}}{\left[\sum_{\mathbf{n}}\left|\phi_{\mathbf{n}}^{s t, \kappa}\right| 2\right]^{2}}, \\
m_{2}=\Delta^{2} \sum_{\mathbf{n}}\left(\left|\phi_{\mathbf{n}}^{s t, \kappa}\right|^{2}\right)^{2}[c+p(\mathbf{n})][1-c-p(\mathbf{n})] .
\end{gathered}
$$

Local values of the attractive and repulsive atom concentrations are described by the expressions $[c+p(\mathbf{n})]$ and $[1$ $-c-p(\mathbf{n})]$, respectively.

The DOS expression for each of the $s$ presents a set of $g_{s t}$ bands, the contours of which are approximately Gaussians near their maxima. The DOS decreases with further increase of energy and transforms for each band into an Urbach exponent. The sum rule in this case can be formulated as an inequality like Eq. (43).

\section{Weak-scattering limit $p_{c}<c<1-p_{c}$}

The weak-scattering regime takes place at all compositions of the solid solution if $\Delta \ll E_{c r}$. The strong-scattering system transforms into weak scattering at compositions for which the inequality $(1-c) \Delta \ll E_{c r}$ holds. A weak fluctuation potential means that the number of localized states is considerably less than the total number of states in the electron band and the tail region is much less than the bandwidth. These facts define the difficulties for the experimental investigation of weak-scattering systems in the region of the tail states.

The $\mathrm{CdS}_{(1-c)} \mathrm{Se}_{c}$ solid solution, which we consider as a weak-scattering system is characterized by a value of the relation $\Delta / E_{c r} \approx 0.3$ for the valence band. When the tailing has its maximum at $c=0.2$ this value means that the critical number of Se atoms necessary to split off a localized state is of the order 10 . The probability of the fluctuation presenting a compact cluster of ten $\mathrm{Se}$ atoms is proportional to $c^{10}$ $\approx 10^{-7}$. This value corresponds to the number of states in the tail equal to $\approx 10^{16}$, which is insufficient to describe the observable tailing effect at this composition. A considerable decrease of tailing occurs in this solid solution in regions both $c>0.50$ and $c<0.10$ but it still remains observable. Therefore, the first problem is to explain the tailing in such kind of solid solution.

At present, there are no direct measurements of $\rho(\omega)$ in the tail region for solid solutions and we perform an estimation of the DOS value in the region of $\omega$ where data on the exciton absorption can be used to verify the accuracy of the calculations. Further, we will find the relation that connects the half-width of the exciton ground-state absorption band and the DOS.

Our calculation of the number of states splitting off the band edge is based on the assumption that the exciton states are localized in fluctuations that are essentially simply connected potential wells. It is supposed that these potential wells are formed by regions where there is an excess of atoms of the narrow-gap component compared to their average concentration. The problem is to find the most probable fluctuations of such kind. The requirements that each well is simply connected, and that the excess concentration in its volume is minimized, leads to an answer that follows from the theory of percolation along the sites of disordered sublattices: the lower bound on the excess concentration is the critical concentration in the percolation problem along the sites of the sublattice. This approach assumes that the fluctuation wells are large in size, and clusters of excess atoms in the wells can be considered as fractals of finite size. The DOS in the tail obtained from these arguments have considerably larger values as compared to estimations of the compact clusters and are in agreement with experimental data over a wide range of concentrations.

\section{Estimation of the quantity of $\rho(\omega)$ for fractal clusters}

We consider the most general case, which involves potential wells in which the excess concentration $p$ of attractive centers averaged over the potential well is smaller than its maximum value $(1-c)$, which leads to a compact occupation of the volume.

The probability of realizing a fluctuation within the volume containing $n_{\omega}$ lattice sites where the excess number of attraction centers is $n_{p}(\omega)=n_{p}=n_{\omega} p$ equals

$$
c^{n_{A}}(1-c)^{n_{B}},
$$


where $n_{A}=n_{\omega}(c+p)=\left(n_{c}+n_{p}\right) \quad$ and $n_{B}=n_{\omega}(1-c-p)$ $=\left(n_{\omega}-n_{c}-n_{p}\right)$ are the numbers of atoms $A$ and $B$ in the considered volume. It is known that the strength of the potential well is only weakly dependent on the form of the well. ${ }^{75} \mathrm{We}$ assume that states with nearly the same localization depth can be obtained if the number $n_{p}$ of excess attractive atoms is comparable to the size $n_{\omega}^{\text {comp }}$ of the compact cluster. Then the probability of realization written above will be considerably smaller than the probability of realization of a compact cluster

$$
\exp \left(n_{\omega}^{c o m p} \ln c\right)=c^{n_{\omega}^{c o m p}}
$$

for approximately the same value of the localization energy. However, taking into account that $n_{\omega} \gg 1$, we obtain for the number of realizations the large quantity

$$
\frac{n_{\omega} !}{n_{A} ! n_{B} !}
$$

which can compensate the small probability for an individual realization.

The contribution to the DOS from clusters of size $n_{\omega}$ containing $n_{p}$ centers of attraction in excess can be written in the form ${ }^{15}$

$$
\begin{aligned}
\rho(\omega)= & \frac{1}{\pi v_{0}}\langle\operatorname{Im} G(\omega)\rangle \\
\approx & \frac{1}{v_{0} \Delta} \sqrt{\frac{n_{\omega}}{2 \pi(c+p)(1-c-p)}} \\
& \times\left(\frac{c}{c+p}\right)^{n_{\omega}(c+p)\left(\frac{1-c}{1-c-p}\right)^{n_{\omega}(1-c-p)}},
\end{aligned}
$$

where $v_{0}=V / N$.

The DOS obtained is strongly decreasing with $p$ increasing and we have to find the wells that give the localized state of a given depth at a minimum value of $p$. The number of excess attractive atoms $A$ in the well is equal to $n_{p}$. These atoms can form a singly connected configuration within a volume containing $n_{\omega} \gg 1$ lattice sites at $p$, which is close to the critical value of site percolation problem $p_{c}$ for a given sublattice. We can define a singly connected potential well of such kind as a finite-size fractal. Then the substitution $p$ $=p_{c}$ into Eq. (51) where $p_{c}$ is the critical value for an infinite sample gives an estimation of the DOS if the actual size of fluctuation is large, i.e., $n_{\omega} \gg 1$.

A further problem consists in the estimation of the localization depth for potential wells. At present there is no recipe for this estimation for potential wells of fractal structure. We perform an estimation based on universal properties of nodeless ground states of the Schrodinger equation in the region of an attractive potential ${ }^{75,76}$ and assume that the form of the potential well plays no role as it takes place for the wells of simple forms. ${ }^{75}$

Let us write the criterion of appearance of the localized state for a compact cluster of spherical form in effectivemass approximation using the approach of Ref. 75, which gives in this case an exact result

$$
\left[n_{c r}^{c o m p}\right]^{2 / 3}(1-c) \Delta=E_{c r},
$$

where $n_{c r}^{c o m p}$ is the size of the potential well, $(1-c) \Delta$ is its depth, and $E_{c r}$ is the critical value in the effective-mass approximation for a potential well with volume $v_{0}$

$$
E_{c r}=\frac{\pi^{2}}{4} \frac{\hbar^{2}}{2 M\left(\frac{3}{4 \pi} v_{0}\right)^{2 / 3}},
$$

where $M$ is the effective mass of the particle. Taking into account the relation between radius $R(\omega)$ of the spherical potential well, its depth $(1-c) \Delta$, the critical value of the single site perturbation of Eq. (53), and the localization energy $\omega$, which has the form

$$
R(\omega)=\sqrt{\frac{\hbar^{2}}{2 M[(1-c) \Delta-\omega]}}\left[\pi-\operatorname{arctg} \sqrt{\frac{(1-c) \Delta-\omega}{\omega}}\right],
$$

we see that Eq. (52) can be withdrawn from the last equation in the limit $\omega \rightarrow 0$. For the potential well that consists of $n_{\omega}$ lattice sites containing $\left(n_{\omega} c\right)$ of attractive atoms and $\left(n_{\omega} p_{c}\right)$ of excess atoms of the same kind, the maximum volume in which the wave function does not decrease exponentially is equal to $n_{\omega}\left(c+p_{c}\right)$. The averaged level of the attractive potential within this volume is equal to $\Delta p_{c} /\left(c+p_{c}\right)$. Then the relation, which connects $n_{\omega}, \omega$, and $E_{c r}$ can be written as

$$
\begin{aligned}
& {\left[n_{\omega}\left(c+p_{c}\right)\right]^{2 / 3}\left[\frac{p_{c}}{c+p_{c}} \Delta-\omega\right]} \\
& \quad=\frac{4 E_{c r}}{\pi^{2}}\left\{\pi-\operatorname{arctg}\left[\sqrt{\frac{p_{c}}{c+p_{c}} \Delta / \omega-1}\right]\right\}^{2} .
\end{aligned}
$$

This leads to the number of lattice sites in the potential well at a given localization energy

$$
\begin{aligned}
n_{\omega}= & \frac{1}{\left(c+p_{c}\right)}\left[\frac{E_{c r}}{\frac{p_{c}}{c+p_{c}} \Delta-\omega}\right]^{3 / 2} \\
& \times\left\{2-\frac{2}{\pi} \operatorname{arctg}\left[\sqrt{\frac{p_{c}}{c+p_{c}} \frac{\Delta}{\omega}-1}\right]\right\}^{3} .
\end{aligned}
$$

The equation obtained gives in the limit $c \rightarrow\left(1-p_{c}\right)$ the exact result for the compact spherical cluster.

Finally, the DOS can be presented in the form

$$
\begin{aligned}
\rho(\omega) \approx & \frac{1}{v_{0} \Delta} \sqrt{\frac{n_{\omega}}{2 \pi\left(c+p_{c}\right)\left(1-c-p_{c}\right)}} \\
& \times\left[\left(\frac{c}{c+p_{c}}\right)^{\left(c+p_{c}\right)}\left(\frac{1-c}{1-c-p_{c}}\right)^{\left(1-c-p_{c}\right)}\right]^{n_{\omega}},
\end{aligned}
$$

with $n_{\omega}$ defined by Eq. (56). We shall use this equation for the estimation of the DOS in the region of the absorption maximum of the exciton ground state. 


\section{Weak-scattering regime in regions $c<p_{c}$ and $c>1-p_{c}$}

The tailing estimated by Eq. (57) decreases sharply at low concentration of attractive atoms $c<p_{c}$ in the weak scattering limit. The $\mathrm{CdS}_{(1-c)} \mathrm{Se}_{c}$ solid solution shows, nevertheless, an observable broadening of the exciton ground state even in this region of concentration. Equation (57) gives the correct order of the DOS in this solid solution at an energy corresponding to the exciton band maximum up to $c=0.1$. At lower concentrations the value $p_{c}=0.2$ leads to a number of states, which is insufficient to explain the observed tailing effects.

To decrease the value of $p_{c}$ and thus to increase the number of localized states in this region of concentration we consider potential wells formation by finite-size clusters taking into account the percolation over the next neighbors.

Another concentration range where the percolation over the next neighbors should be taken into account is $c>1$ $-p_{c}$. In this composition region the localized states can be isolated from each other only if the repulsive atoms form a percolation cluster, which in turn demands a decreasing percolation threshold.

\section{E. Approximation of the energy dependence of the density of states}

The following calculations are aimed to obtain an interpolation formula, which describes the dependence of DOS in the wide region of energies for both strong- and weakscattering limits. It is assumed that $\omega_{0}$ is the lower border of the energy interval where the DOS behavior is considered. We assume that in zeroth approximation all the states with localization energy $\omega_{j}>\omega_{0}$ can be treated as localized and isolated from the others. This approach is valid if the total number of states with $\omega>\omega_{0}$

$$
\mathcal{N}\left(\omega_{0}\right)=\int_{\omega_{0}}^{E_{L}} \rho(\omega) d \omega
$$

satisfies the inequality

$$
\mathcal{N}\left(\omega_{0}\right) a^{3}<1
$$

Here $a \approx \sqrt{\hbar^{2} / 2 M \omega_{0}}$ is the typical length of the exponential decay of the wave function with a localization energy of the order $\omega_{0}$. The further consideration will show that $\omega_{0}$ $<\omega_{M E}$ where $\omega_{M E}$ is position of the mobility edge. Both these energies have the same order of magnitude and are considerably larger then the Urbach parameter $\varepsilon_{U}$, which defines the exponential decrease of the DOS in the region of energies exceeding $\omega_{M E} \cdot{ }^{77}$

We describe the behavior of $\rho(\omega)$ in effective-mass approximation using modifications of the variational procedure of Refs. 78 and 79 and assuming that localized states are formed by the spherical potential wells, which are larger than the compact cluster leading to the localized state at energy $\omega_{0}$. Using the approach developed in Refs. 14 and 15 we can write for the DOS

$$
\begin{aligned}
\rho(\omega) \sim & \frac{1}{v_{0} \sqrt{2 \pi \gamma^{2}(\omega)}} \exp \left\{\int \frac{d^{3} r}{v_{0}}\right. \\
& \left.\times\left[\ln \left(\frac{c}{c+p(r)}\right)^{c+p(r)}\left(\frac{1-c}{1-c-p(r)}\right)^{1-c-p(r)}\right]\right\},
\end{aligned}
$$

where

$$
\gamma^{2}(\omega)=\frac{m_{2}}{\left[\int \frac{d^{3} r}{v_{0}} \varphi_{t r}^{2}(r)\right]^{2}}
$$

and

$$
m_{2}=\Delta^{2} \int \frac{d^{3} r}{v_{0}}\left[\varphi_{t r}^{2}(r)\right]^{2}[c+p(r)][1-c-p(r)] .
$$

The local values of attractive and repulsive atom concentrations are described by the equations $[c+p(r)]$ and $[1-c$ $-p(r)]$, respectively. The potential well configuration $U_{t r}(r)$ is given by the function $p(r)$

$$
U_{t r}(r)=-\Delta p(r),
$$

where

$p(r)$

$$
=\left\{\begin{array}{l}
(1-c) \quad r \leqslant R\left(\omega_{0}\right) \\
(1-c)\left[1-\frac{\exp \left(-t \varphi_{t r}^{2} \Delta\right)}{c+(1-c) \exp \left(-t \varphi_{t r}^{2} \Delta\right)}\right] \quad r>R\left(\omega_{0}\right)
\end{array}\right\},
$$

and $R\left(\omega_{0}\right)$ is expressed by Eq. (54). The trial function $\varphi_{t r}(r)$ is the solution of the equation

$$
\left[-\frac{\hbar^{2}}{2 M} \nabla^{2}+\omega-U_{t r}(r)\right] \varphi_{t r}(r)=0
$$

The DOS obtained in this way decreases slowly at values of $\omega$, which are not too large as compared to $\omega_{0}$ and it has singular behavior at $\omega \rightarrow E_{L}$, i.e., near the Lifshitz border. This character of the DOS behavior corresponds well to the energy dependence of the experimental data, however, the absolute number of the states appears to be insufficient to describe the tailing. This occurs because of the fact that the procedure takes into account only the localized states, which are formed in spherical potential wells while in reality there exists a great variety of their space configurations. We introduce a multiplication factor that corrects the magnitude of $\rho(\omega)$ of Eq. (60) using the sum rule of Eq. (43) in the case of the strong scattering limit and estimations of the number of localized states in the region of absorption band maximum given by Eq. (57).

\section{F. Zero-phonon exciton absorption band}

For an ideal crystal the optical absorption is described in the wavelength region of the ground-state exciton by the spectral density, which can be written in the form 


$$
\alpha_{1 s}(\omega) \sim \frac{v_{0}}{\pi}\left|\psi_{1 s}(0)\right|^{2} \operatorname{ImG}_{00}\left(\omega-E_{1 s}-i \delta\right),
$$

where

$$
G_{\vec{k} \vec{k}}\left(\omega-E_{1 s}\right)=\frac{1}{\omega-\frac{\hbar^{2}}{2 M} \vec{k}^{2}-E_{1 s}-i \delta}
$$

Here $E_{1 s}$ and $\psi_{1 s}(r) \sim \exp \left(-r / a_{B}\right)$ are the eigenvalue and eigenfunction of the exciton ground state, and $k$ is the wave vector of a photon.

For the solid solution the general form of the imaginary part of the Green's function averaged over possible realizations of the fluctuation potential is ${ }^{77}$

$$
\left\langle\operatorname{ImG}_{\vec{k} \vec{k}}(\omega)\right\rangle=\frac{\tau_{\vec{k} \vec{k}}(\omega)}{\left[\omega-\frac{\hbar^{2}}{2 M} \vec{k}^{2}-\Delta_{\vec{k} \vec{k}}(\omega)\right]^{2}+\tau_{\vec{k} \vec{k}}^{2}(\omega)},
$$

where $\tau_{\vec{k} k}(\omega)$ and $\Delta_{\vec{k} \vec{k}}(\omega)$ are linked by the dispersion relation, which ensures correct normalization of the expression for the spectral density. In the region of localized states these functions are the imaginary and real parts of the scattering matrix. ${ }^{75}$ Therefore, we have

$$
\tau_{\vec{k} \vec{k}}(\omega)=\frac{6 v_{0}}{\pi^{3}} \Delta^{2} \sqrt{(\omega / \Delta)}\left(\frac{E_{c r}}{\Delta}\right)^{3 / 2}\left|I_{1}(\vec{k})\right|^{2} \rho(\omega),
$$

where

$$
I_{1}(\vec{k})=\left(\frac{2 M \omega}{\hbar^{2}}\right)^{3 / 2} \frac{1}{(2 \pi)^{3}} \int d^{3} \vec{r} \exp (i \vec{k} \vec{r}) \varphi_{t r}(\vec{r})
$$

The calculation of $\Delta_{\vec{k} k}(\omega)$ is a more complicated problem because for this purpose we have to know $\tau_{\vec{k}} \vec{k}(\omega)$ in a wideenergy interval.

For an approximate description of the spectral density within a narrow energy region in the vicinity of its maximum we can replace $\Delta_{\vec{k} k}(\omega)$ by a constant value $\Delta_{\vec{k} \vec{k}}^{0}$. As the result we obtain at $k \approx 0$

$$
\left\langle\operatorname{ImG}_{00}(\omega)\right\rangle \approx \frac{1}{\pi\left[\omega-\Delta_{00}^{0}\right]^{2}+\tau_{00}^{2}(\omega)},
$$

where $\Delta_{00}^{0}$ is considered as a parameter, which allows us to reach coincidence between the positions of experimental and theoretical maxima $\omega_{\max }$ of the $1 s$ exciton state in the absorption spectrum.

\section{G. Zero-phonon luminescence band}

Until now we considered the states with energies $\omega \geqslant \omega_{0}$ as isolated from each other. These states are formed by clusters of atoms of the narrow-gap component of the solid solution or by singly connected potential wells, which are induced by composition fluctuations. At random distribution of the constituents of the solid solution the clusters and potential wells are also randomly distributed over the crystal. Now we introduce a more accurate classification of the states taking into account that not all of them are really isolated in space. In general, the consideration of randomly distributed potential wells, the wave functions of which are overlapping, is a sophisticated variant of the problem of quantum percolation theory ${ }^{80-86}$ with off-diagonal disorder. We simplify the problem and apply for the consideration a continuum version of classical percolation theory.

\section{Classification of the tail states}

The problem that we intend to solve is whether or not a given state has the possibility for a transition to any other lower laying states followed by emission of phonons. We suppose that the temperature is low enough to make transitions into upper states unimportant. For this purpose we introduce a sphere of radius $R_{\text {int }}$, which restricts the length of the step that can be made in processes of such electronphonon relaxation. These processes are completely absent for the deeply localized states for any reasonable value of $R_{i n t}$. Because of the exponential increase of the DOS with decreasing $\omega$ the role of these processes grows and becomes critical within a relatively narrow region of energies. This fact allows one to consider $R_{\text {int }}$ as an energy independent parameter. As a result the problem of the description of the tail states can be considered in terms of continuum percolation theory for overlapping spheres. ${ }^{16-22}$ The percolation theory allows introduction of subdivision of the tail states into states formed by spatially isolated clusters (or potential wells), by complexes of clusters (or potential wells), and states belonging to percolation clusters.

We consider a state with localization energy $\omega$ as spatially isolated if there exist no states with larger localization energy in the sphere of radius $R_{\text {int }}$ surrounding the given potential well. The number of isolated states $\left\langle n_{1}\right\rangle$ can be written with the help of the continuum percolation theory for overlapping spheres ${ }^{16-21}$ as

$$
\left\langle n_{1}(\omega)\right\rangle \approx \exp \left(-\left[R_{\text {int }} / \overline{r(\omega)}\right]^{3}\right) \equiv \exp \{-2 \mathcal{P}(\omega)\} .
$$

Here notations are introduced

$$
\mathcal{P}(\omega)=\frac{1}{2}\left[R_{\text {int }} / \overline{r(\omega)}\right]^{3}, \quad \overline{r(\omega)}=\left(\frac{3}{4 \pi} \frac{1}{\mathcal{N}(\omega)}\right)^{(1 / 3)} .
$$

The integrated DOS $\mathcal{N}(\omega)$ is given by equation

$$
\mathcal{N}(\omega)=\int_{\omega}^{E_{L}} \rho(\omega) d \omega
$$

The function $\mathcal{P}(\omega)$ represents the integrated density of localized states with localization energy exceeding $\omega$ in units of the first virial coefficient.

We consider the state as belonging to a supercluster of size $s$ if it is connected with any others of $(s-1)$ states with larger localization energy, i.e., if there exist paths from the given state to the other $(s-1)$ states, which can be made by steps that are less than $R_{\text {int }}$. Then the magnitude of $\mathcal{N}(\omega)$ reaches the critical value at certain localization energy $\omega_{M E}$ and a supercluster of infinite size appeares. This supercluster is regarded as percolation cluster and $\omega_{M E}$ is considered as the mobility edge. 
We shall use the fact that the critical value of the density for the percolation problem for overlapping spheres is known. Survey of data on critical values obtained by a number of authors are given by Haan and Zwanzig. ${ }^{20}$ These values lay within the interval $1.17 \leqslant \mathcal{P}_{c r}^{c l} \leqslant 1.40$. It follows from Eqs. (72) and (73) that the critical value in our problem $\mathcal{P}\left(\omega_{M E}\right)$ depends on the magnitude of $R_{i n t}$ and on the integrated DOS at the percolation threshold $\mathcal{N}\left(\omega_{M E}\right)$.

The important characteristics of the percolation theory of overlapping spheres ${ }^{16-22}$ are the averaged numbers of clusters $\left\langle n_{s}\right\rangle$ consisting of $s$ overlapping spheres. We identify these values with numbers of superclusters formed by the potential wells. The numbers $\left\langle n_{s}\right\rangle$ were obtained for the first five $s$ in the low-density limit by Haan and Zwanzig ${ }^{20}$ in the form of a Taylor series. In order to estimate the behavior of $\left\langle n_{s}\right\rangle$ in the region of the mobility edge we have performed an extrapolation of the dependences for $s=2,3,4$ by means of equations

$$
\begin{gathered}
\left\langle n_{2}(\omega)\right\rangle=\mathcal{P}(\omega) \exp \{-3.073 \mathcal{P}(\omega)\}, \\
\left\langle n_{3}(\omega)\right\rangle=1.375 \mathcal{P}^{2}(\omega) \exp \{-4.09 \mathcal{P}(\omega)\}, \\
\left\langle n_{4}(\omega)\right\rangle=2.1842 \mathcal{P}^{3}(\omega) \exp \{-5.084 \mathcal{P}(\omega)\} .
\end{gathered}
$$

The Taylor series expansion of these functions gives results practically coinciding with those of Haan and Zwanzig. ${ }^{20}$ The accuracy of the extrapolation of the $\left\langle n_{2}(\omega)\right\rangle$ can be examined by using the results of numerical calculations of Holcomb and Rehr ${ }^{18}$ for the function $P_{3}\left(p_{n o r m}\right)$, which is related with $\left\langle n_{2}(\omega)\right\rangle$ by the equation $P_{3}\left(p_{n o r m}\right)=1-\left\langle n_{1}\right\rangle$ $-2\left\langle n_{2}\right\rangle$ where $p_{\text {norm }}$ and $\mathcal{P}(\omega)$ are connected by the equation $p_{\text {norm }}=\mathcal{P}(\omega) / 8$. The function $P_{3}\left(p_{\text {norm }}\right)$ gives the probability for any sphere to belong to cluster of size $s>3$. The comparison has shown that Eq. (75) leads for $n_{2}$ to close coincidence with results of Holcomb and Rehr ${ }^{18}$ in the region most important for further calculations $p_{\text {norm }}>0.1$, i.e., in the mobility edge region.

All of the states contribute to the exciton ground-state absorption while the steady-state luminescence band at low intensity of excitation is supposed to be formed only by the "radiative" states of the fluctuation tail. The maximum of the "radiative", state density is below the mobility edge and, therefore, the luminescence band decreases sharply in the region of the mobility edge.

\section{Lifetimes of the tail states}

Now it is possible to find the number of states that have no ways for transitions into lower-lying states and have to disappear by means of radiative annihilation. This quality should be attributed first of all to the isolated potential wells with $s=1$. Their number is given by Eq. (72). Besides these states the ground states of superclusters with $s>1$ have also only the radiative way to disappear. As a result we can write for the number of "radiative" states, i.e., for the states that are characterized by the radiative lifetime $\tau^{(0)} \equiv \tau_{\text {rad }}$

$$
\mu_{0}(\omega) \equiv \mu_{\text {rad }}(\omega)=\left\langle\sum_{s=1}^{\infty} n_{s}(\omega)\right\rangle
$$

Here $n_{s}(\omega)$ is the concentration of superclusters consisting of $s$ potential wells with localization energy exceeding $\omega$. The averaging procedure over all possible realizations of superclusters is denoted as $\langle\ldots\rangle$.

Within the considered model all the states of superclusters except the ground state should be characterized by lifetimes resulting from exciton-phonon transitions. Let us enumerate the states of superclusters in order of decreasing localization energy. Then the second state of superclusters with $s=2$ and the second states of all larger superclusters will have as their only decay channel transitions into a corresponding ground state. With increasing supercluster state number the number of channels for exciton-phonon decay processes increases as well. We assume that the lifetime depends only on the number of decay channels. Then the state with number $s>1$ should be characterized by lifetime

$$
\tau^{(s-1)}=\frac{\tau_{d e c}^{(s-1)} \tau_{\text {rad }}}{\tau_{d e c}^{(s-1)}+\tau_{\text {rad }}},
$$

where the index $(s-1)$ is equal to the number of decay channels and $\tau_{d e c}^{(s-1)}$ is the lifetime or intraband relaxation time via exciton-phonon transitions into lower-lying states. The number of states that have $s$ decay channels can be written in analogy with Eq. (76) as

$$
\mu_{s}(\omega)=\left\langle\sum_{k=s+1}^{\infty} n_{k}(\omega)\right\rangle .
$$

The value of $\mu_{\text {rad }}$ can be presented in a first approximation as

$$
\begin{aligned}
\mu_{\text {rad }} & \approx\left\langle n_{1}(\omega)+n_{2}(\omega) \cdots\right\rangle \\
& =\exp [-2 \mathcal{P}(\omega)]+\mathcal{P}(\omega) \exp [-3.073 \mathcal{P}(\omega)] \cdots,
\end{aligned}
$$

while for the fraction of the states that have the single-decay channel $\mu_{1}$ we have

$$
\begin{aligned}
\mu_{1} \approx & \left\langle n_{2}(\omega)+n_{3}(\omega)+\cdots\right\rangle=\mathcal{P}(\omega) \exp [-3.073 \mathcal{P}(\omega)] \\
& +1.375 \mathcal{P}^{2}(\omega) \exp [-4.09 \mathcal{P}(\omega)]+\cdots .
\end{aligned}
$$

Sums like the ones given by Eqs. (76) and (78) can be estimated by their lower limit ${ }^{22}$ with high accuracy, i.e., by their few first terms, which are presented in Eqs. (79) and (80). These sums are well defined both below and within the critical region $|\delta \mathcal{N}(\omega)| \equiv\left|1-\mathcal{N}(\omega) / \mathcal{N}\left(\omega_{M E}\right)\right| \ll 1$, and above that. However, they are exponentially small in the last two regions. Using the approach of Ref. 22 it is possible to estimate the singular part of these sums in the critical region as $|\delta \mathcal{N}(\omega)|^{(\nu D+1)}$, where $D$ is the dimensionality of the system and $\nu$ is the critical index of the order parameter depending on the dimensionality of the system. For $D=3$ the value of $\nu$ is approximately 0.875 , which leads to negligibly small values of the singular parts of these sums in the critical region.

\section{H. Shape of the zero-phonon luminescence band at low intensity of excitation}

The calculation of the shape of the zero-phonon luminescence band will be performed at the condition that the optical 


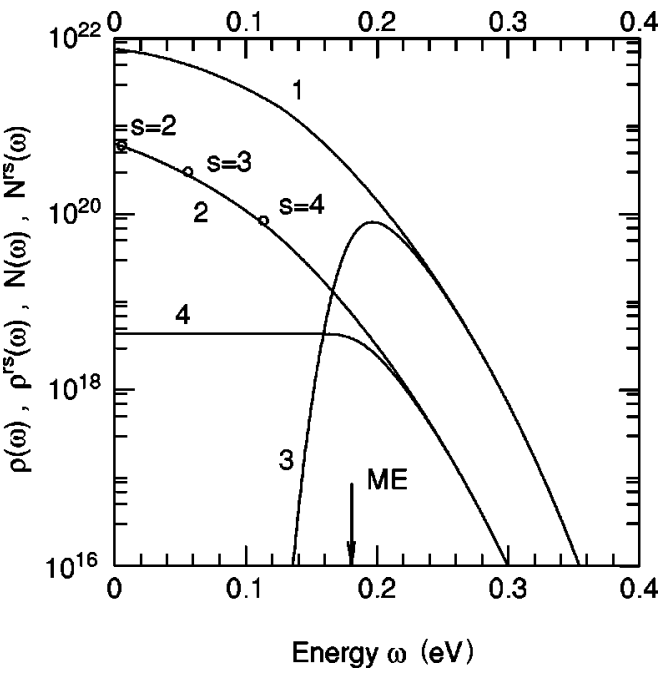

FIG. 1. Density of states $\rho(\omega)(1)$, integrated density of states $\mathcal{N}(\omega)(2)$, density of "radiative", states $\rho^{r s}(\omega)$ (3), and integrated density of "radiative" states $\mathcal{N}^{r s}(\omega)$ (4) of the fluctuation tail of the exciton ground state in the solid solution $\mathrm{ZnSe}_{1-c} \mathrm{Te}_{c}$ at $c$ $=0.13$. Curves (1) and (3) are represented in the units $\left[\mathrm{cm}^{-3} \mathrm{eV}^{-1}\right]$, curves (2) and (4)-in $\left[\mathrm{cm}^{-3}\right]$. Open circlersestimation of integral density of states using the sum rule Eq. (43). Estimated position of the mobility edge $\omega_{M E}$ is indicated by the arrow.

recombination is restricted by the nonradiative transitions into the lower-lying localized tail states.

The steady-state density of the populated states at weak interband excitation is proportional to the product of the DOS at a given energy and the lifetime of the state. The radiation probability of the populated states is described by the same optical DOS as the absorption coefficient $\alpha^{0}(\omega)$. Therefore, the zero-phonon luminescence band can be described as

$$
I_{1 s}^{0}(\omega) \sim \alpha_{1 s}^{0}(\omega) P(\omega) \tau_{\text {rad }},
$$

where $P(\omega)$ is the total relative fraction of the radiative states at energy $\omega$, which belongs to superclusters of different size.

$$
P(\omega)=\sum_{s=1}^{\infty} \mu_{(s-1)}(\omega) \frac{\tau^{(s-1)}}{\tau_{\text {rad }}} \equiv \sum_{s=1}^{\infty} P^{(s-1)}(\omega) .
$$

The contribution to $P(\omega)$ of isolated wells and of ground states of two-well superclusters is given by the equation

$$
P(\omega) \sim\{\exp [-2 \mathcal{P}(\omega)]+\mathcal{P}(\omega) \exp [-3.073 \mathcal{P}(\omega)]\}
$$

We have accepted in our calculations a critical value for the $\mathcal{P}\left(\omega_{M E}\right)=1.40$ taken from Ref. 20. The first correction to Eq. (83) can be written as

$$
\begin{aligned}
& \alpha_{1 s}^{0}(\omega) P^{(1)}(\omega) \tau_{\text {rad }} \\
& \quad \approx \alpha_{1 s}^{0}(\omega)\left\langle n_{2}(\omega)\right\rangle \tau^{(1)} \\
& \quad=\alpha_{1 s}^{0}(\omega) \mathcal{P}(\omega) \exp [-3.073 \mathcal{P}(\omega)] \frac{\tau_{d e c} \tau_{r a d}}{\tau_{d e c}+\tau_{r a d}} .
\end{aligned}
$$

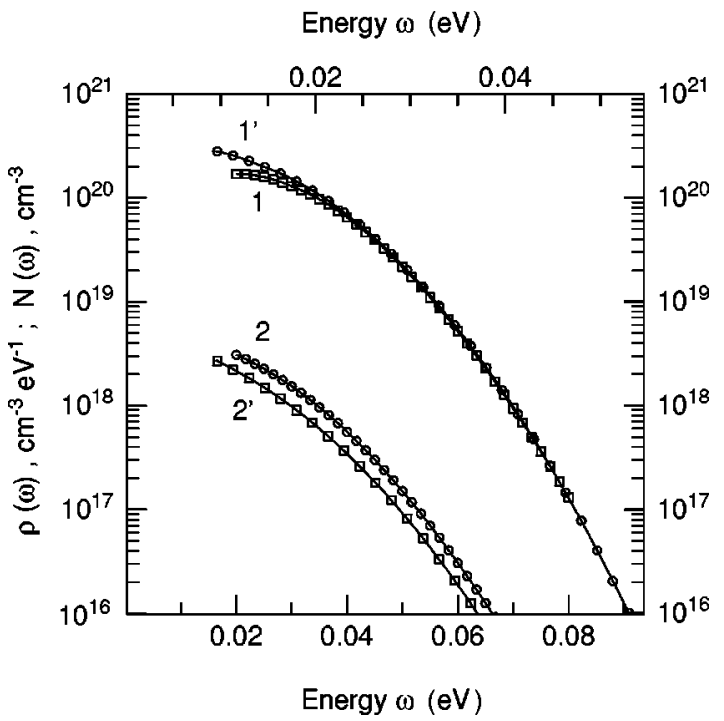

FIG. 2. Density of states $\rho(\omega)\left(1,1^{\prime}\right)$ and integrated density $\mathcal{N}(\omega)\left(2,2^{\prime}\right)$ of the fluctuation tails of the exciton ground state in the solid solution $\mathrm{CdS}_{1-c} \mathrm{Se}_{c}$ at $c=0.2(1,2)$ (lower energy scale) and $c=0.51\left(1^{\prime} 2^{\prime}\right)$ (upper energy scale).

Here we denoted as $\tau_{d e c}$ the lifetime resulting from the decay process for the first "excited" state of the pair supercluster. The last equation shows that the role of the correction depends on the relation between $\tau_{d e c}$ and $\tau_{\text {rad }}$.

The isolated potential wells and superclusters of small size give the major contribution to the luminescence at all energies of interest while the superclusters of higher order give small corrections only.

Figures 1, 2, and 3 demonstrate the results of model calculations for the strong and weak scattering, respectively.

Figure 1 presents the DOS of the fluctuation tail states $\rho(\omega)$ and the integrated DOS $\mathcal{N}(\omega)$ together with the DOS of "radiative" states calculated by means of equation

$$
\rho^{r s}(\omega)=\rho(\omega) P(\omega),
$$

where $P(\omega)$ is given by Eq. (83). The corresponding integrated DOS $\mathcal{N}^{r s}(\omega)$ of "radiative" states is also shown in Fig. 1 for the $\mathrm{ZnSe}_{(1-c)} \mathrm{Te}_{c}$ solid solution at $c=0.13$. For completeness, the position of the mobility edge $\omega_{M E}$ is presented in Fig. 1 as a vertical arrow. It is obtained as the result of the fitting of the shape of the luminescence band.

The value of $\omega_{0}$ was taken to be equal to $0.0975 \mathrm{eV}$ in the numerical calculations. This value lays between the estimated localization energies of Te clusters with numbers $s$ $=3$ and 4 . The evaluations of the integrated DOS with the help of the sum rule Eq. (43) are presented in Fig. 1 for $s$ $=2,3$, and 4 as well. The calculation of the DOS was performed in the range $\omega \geqslant \omega_{0}$ using Eq. (60). The obtained curve was corrected with the help of the multiplication factor as it is described above in order to reach coincidence between the integrated DOS and its estimation given by Eq. (43) at $\omega \approx \omega_{0}$. The extrapolation of the DOS into the region of $\omega \leqslant \omega_{0}$ given in Fig. 1 was performed also with the help of Eq. (43). The data of Fig. 1 show that the tailing has in this solid solution a considerable extent and the width of the region of radiative states reaches tens of meV. 


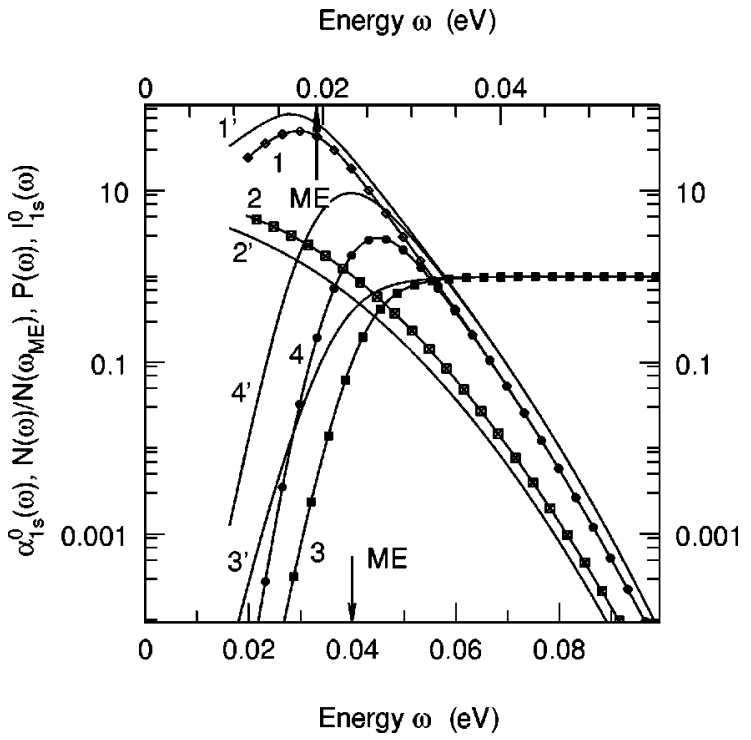

FIG. 3. Zero-phonon absorption of the exciton ground state $\alpha_{1 s}^{0}(\omega)$ in the solid solution $\mathrm{CdS}_{1-c} \mathrm{Se}_{c}(1)$, relative integral density of states $\mathcal{N}(\omega) / \mathcal{N}\left(\omega_{M E}\right)$ (2), fraction of "radiative" states $P(\omega)$ (3) and zero-phonon luminescence band $I_{1 s}^{0}(\omega)(4), c=0.2$ (lower energy scale). Curves $1^{\prime}-4^{\prime}$ are the same for $c=0.51$ (upper energy scale). The arrows indicate the estimated position of the mobility edge $\omega_{M E}$ for these two concentrations.

In Fig. 2 we present the DOS and the integrated DOS calculated for $\mathrm{CdS}_{(1-c)} \mathrm{Se}_{c}$ at $c=0.20$ and 0.51. The curves presenting both these functions look very similar for two different concentrations after a scale transformation of the energy axis. We have used in both cases the estimation of the DOS near the absorption band maximum in accordance with Eq. (57).

Figure 3 shows the zero-phonon optical densities for the absorption and for the luminescence for two compositions of $\mathrm{CdS}_{(1-c)} \mathrm{Se}_{c}$. The curves were obtained with the help of Eqs. (71) and (81). The position of $\omega_{M E}$ was chosen to reach agreement with the experimental shift of the luminescence bands with respect to the absorption spectra. ${ }^{4}$

The shapes of the calculated zero-phonon luminescence bands are defined in the region of energies $\omega>\omega_{M E}$ by the universal Urbach dependence of $\alpha_{1 s}(\omega)$, by the dependence of $\mathcal{N}(\omega)$, which has also Urbach-like character, and by the equations of the percolation theory, which define the behavior of $P(\omega)$. This region of energies includes about $80-90 \%$ of the integrated intensity of the luminescence.

A more complicated character is inherent to the behavior of the zero-phonon luminescence band in the interval $\omega_{0}$ $<\omega \leqslant \omega_{M E}$. The behavior looks like exponential with a parameter of the slope, which can be expressed as $\left(\left\{\ln \left[I_{1 s}^{0}(\omega)\right]\right\}_{\omega}^{\prime}\right)^{-1}$. This value is well observable in experiments. ${ }^{8}$ The latter region of energies is characterized by $P(\omega)$ values, which are considerably less than unity, and which decrease rapidly with decreasing $\omega$. The optical density of "radiative" states consists of only a small fraction of the total optical density of the exciton band in this region. Therefore, we can expect that this region of the luminescence spectrum can be changed even by a low population of the nonradiative ("silent") states at a relatively weak excitation.

\section{Luminescence of "silent" states}

The main assumption of our previous consideration is that the main part of states optically active in absorption remains "silent" in luminescence under low-power steady-state excitation. The situation can be changed at high excitation when a considerable fraction of the radiative states is populated, and therefore a significant fraction of the states attributed as "nonradiative"' can be populated too. The resulting additional zero-phonon luminescence can then be described as follows

$$
\delta I_{1 s}^{0}(\omega) \sim \alpha_{1 s}^{0}(\omega) f\left[\omega, \tau(\omega) / \tau_{\text {rad }}\right][1-P(\omega)] \tau_{\text {rad }},
$$

where the function $f\left[\omega, \tau(\omega) / \tau_{\text {rad }}\right]$ describes the population level of the nonradiative states at energy $\omega$, which are characterized by their lifetime $\tau(\omega)$. The function $f\left[\omega, \tau(\omega) / \tau_{\text {rad }}\right]$ as well as $\tau(\omega)$ depends on the intensity of excitation. The fraction of nonradiative states is given by the function $[1-P(\omega)]$, which at $\omega \leqslant \omega_{M E}$ satisfies the inequality $[1-P(\omega)] \gg P(\omega)$ because of the exponential decrease of $P(\omega)$ in this region. The spectral range where the additional luminescence $\delta I_{1 s}^{0}(\omega)$ could appear is above the region of the maximum of $I_{1 s}^{0}(\omega)$. This fact means that the emission of usually "silent" states will change the position of the maximum and the short wave wing of the steady-state luminescence band.

It is worth noting that the role of the additional emisson described by Eq. (86) depends at any given intensity of excitation on the total number of tail states. All other conditions being equal, the population of the "silent" states are more important for the concentrations of the solid solution for which the number of tail states is relatively small. The recombination spectrum can be affected in the cases of (i) weak scattering at low concentrations of the attractive component and (ii) in both weak- and strong-scattering limits at high concentrations of the attractive component. In both cases the number of tail states is not large enough and the radiative states can be saturated even at a relatively low intensity of excitation.

\section{J. Exciton-phonon interaction}

The final stage of calculations includes the excitonphonon interaction. General equations can be presented as

$$
\alpha_{1 s}(\omega)=\int_{0}^{\infty} d z \alpha_{1 s}^{0}(\omega+z) F(z)
$$

and

$$
I_{1 s}(\omega)=\int_{-\infty}^{0} d z I_{1 s}^{0}(\omega-z) F(z)
$$

When using well-known results ${ }^{87,88}$ we can write the density of the phonon wing for localized exciton at the temperature $T=0$ as 


$$
\begin{aligned}
F(\omega)= & \frac{1}{2 \pi} \int_{-\infty}^{\infty} d t \exp \left[i \omega t+\sum_{q} \frac{\left|H_{\Phi \Phi}(\vec{q})\right|^{2}}{\Omega_{q}^{2}}\right. \\
& \left.\times\left[\exp \left(-i \Omega_{q} t\right)-1\right]\right] .
\end{aligned}
$$

Our purpose is to obtain the absorption coefficient and the luminescence intensity as functions of the localization energy taking into account the diagonal exciton-phonon interaction with both LO and LA phonons when no change of the excitonic state occurs. Equations (71) and (81) describe the inhomogeneous zero-phonon band. In order to calculate the matrix elements of the Hamiltonian of the exciton-phonon interaction $H_{\Phi \Phi}(\vec{q})$ the wave function of the localized excitons can be written as

$$
\Phi=\phi_{t r}(\vec{R}) \psi_{1 s}(\vec{r}) .
$$

Here $\phi_{t r}$ is the solution of Eq. (65) and $\psi_{1 s} \sim \exp \left(-r / a_{B}\right)$ is the wave function of the exciton ground state with Bohr radius $a_{B}$.

The considerable difference appears in the excitonphonon interaction for two models of localized excitons: (I) excitons trapped as a whole and (II) the fluctuation trapped hole with Coulomb bound electron.

The argument $\vec{R}$ of $\phi_{t r}$ is the center-of-mass vector in the model I and it coincides with the hole coordinate $\vec{R}=\overrightarrow{r_{h}}$ in model II. The argument $\vec{r}$ of $\psi_{1 s} \vec{r}=\overrightarrow{r_{h}}-\overrightarrow{r_{e}}$ in model I and it is equal to the electron coordinate $\vec{r}=\vec{r}_{e}$ for model II.

The Hamiltonian $H^{f}$ is given by the sum

$$
H_{L O}^{f}+H_{L A}^{f},
$$

where $f$ labels the phonon states. Each of the terms of this equation in turn presents the sum of the electron and hole Hamiltonians

$$
H_{L O, L A}^{f}=H_{L O, L A}^{e}+H_{L O, L A}^{h} .
$$

The matrix elements of the exciton-phonon Hamiltonian can be presented in the form ${ }^{3,89}$

$$
H_{\Phi \Phi}^{\alpha, f}=\left[F_{e, q}^{\alpha} \exp i\left(\vec{q} \vec{r}_{e}\right)+F_{h, q}^{\alpha} \exp i\left(\vec{q} \vec{r}_{h}\right)\right]_{\Phi \Phi} .
$$

Taking into account that for the model I

$$
\overrightarrow{r_{e, h}}=\vec{R}+\frac{\mu}{m_{h, e}} \vec{r} ; \quad \vec{r}=\overrightarrow{r_{e}}-\overrightarrow{r_{h}} ; \quad \mu=m_{e} m_{h} /\left(m_{e}+m_{h}\right),
$$

we see that for the model I the matrix element of the Hamiltonian can be written as

$$
\begin{aligned}
H_{\Phi \Phi}^{\alpha, f}= & {[\exp i(\vec{q} \vec{R})]_{\phi_{t r} \phi_{t r}}\left\{F_{e, q}^{\alpha}\left[\exp i\left(\vec{q} r \frac{\mu}{m_{h}}\right)\right]_{1 s 1 s}\right.} \\
& \left.+F_{h, q}^{\alpha}\left[\exp i\left(\vec{q} r \frac{\mu}{m_{e}}\right)\right]_{1 s 1 s}\right\} .
\end{aligned}
$$

The first matrix element can be calculated numerically while the calculation of those in square brackets can be done analytically ${ }^{3,89}$ with the help of the exciton ground-state wave function.
For the model II each of the exponents depends only on one argument, and therefore

$$
H_{\Phi \Phi}^{\alpha, f}=F_{e, q}^{\alpha}\left[\exp i\left(\vec{q} \vec{r}_{e}\right)\right]_{1 s 1 s}+F_{h, q}^{\alpha}\left[\exp i\left(\vec{q} \vec{r}_{h}\right)\right]_{\phi_{t r} \phi_{t r}} .
$$

Here the first matrix element can be evaluated analytically. The functions $F_{e, q}^{\alpha}$ and $F_{h, q}^{\alpha}$ are given by Cohen and Sturge $^{3}$ for the Fröhlich interaction $(\alpha=F)$, for the deformation $(\alpha=D)$, and for piezoelectric $(\alpha=P)$ coupling of acoustical phonons.

In order to obtain masses of particles, exciton binding energies, and the exciton-phonon coupling parameters for alloys at arbitrary compositions we have used the procedure of linear interpolation of those for pure crystals from Ref. 90 as it is described in Ref. 3.

\section{EXPERIMENTAL RESULTS AND DISCUSSION}

\section{A. Experimental details}

\section{Samples}

For the investigation of luminescence properties of the mixed crystals $\mathrm{CdS}_{1-c} \mathrm{Se}_{c}$ and $\mathrm{ZnSe}_{1-c} \mathrm{Te}_{c}$ we studied several samples of each system. For simplicity we present in the following data from two representative, high-quality samples of each solid solution. The samples of the $\mathrm{CdS}_{1-c} \mathrm{Se}_{c}$ system, which crystallizes in wurtzite structure over the whole composition range, were an epitaxial layer and a thin single crystal platelet with slightly different Selenium contents of $5 \%$ and $7 \%$, respectively. The platelet sample was grown from the gas phase, the crystalline $\vec{c}$-vector being parallel to the surface. The epitaxial layer was grown by hot wall epitaxy (HWE) on the [111] surface of a GaAs substrate. Its $\vec{c}$-axis is oriented perpendicular to the surface, along the epitaxial growth direction. For details of the growth procedure and the characterization of the sample see Ref. 25. The studied samples of the zinc-blende solid solution $\mathrm{ZnSe}_{1-c} \mathrm{Te}_{c}$ were bulk crystals with Tellurium contents of $13 \%$ and $10 \%$. They were grown from the melt but at slightly different growing temperature and excess Te vapor pressure.

\section{Steady-state and low-intensity pulse excitations}

To study the properties of luminescence and its dynamics, we carried out experiments under steady-state conditions and under pulsed excitation combined with time-resolved detection. For the $\mathrm{cw}$ characterization of the samples we used the UV lines of a mercury lamp or an attenuated line of an $\mathrm{Ar}^{+}$ ion laser as excitation source. In order to investigate the luminescence dynamics under low and high excitation, we performed measurements with two different experimental setups, which provide $p s$ pulses with high-repetition rates at low-pulse energies and pulses with high-pulse power but low repetition, respectively. In both cases we were able to tune the wavelength of the exciting light from above the band edge to resonant excitation into the localized states. The first setup consisted of an $\mathrm{Ar}^{+}$ion pumped passive mode-locked Ti:Sa laser followed by a regenerative amplifier and an optical parametric amplifier for second harmonic generation. The spectral sharp pulses had a duration of $\tau_{F W H M} \leqslant 1 p s$ 
with $200 \mathrm{kHz}$ repetition rate and up to $0.1 \mathrm{~mW}$ average excitation power which gives about $2 \mu \mathrm{J} / \mathrm{cm}^{2}$ for density of excitation at the spot diameter $0.2 \mathrm{~mm}$.

The detection of the luminescence signal was done with time-resolved photon counting after the monochrometer. Its temporal resolution was around $200 \mathrm{ps}$. The system allowed to follow the decay of the signal up to $0.5 \mu s$ over a dynamical range of more than four orders of magnitude.

\section{High-intensity pulse excitations}

For the creation of high carrier densities we used an amplified, quenched cavity dye laser pumped by a $\mathrm{XeCl}$ excimer laser. It gives pulses with $\tau_{F W H M}=80 \ldots 120$ ps duration. The maximum excitation intensities used in experiments discussed below was up to $0.1 \mathrm{~mJ} / \mathrm{cm}^{2}$ per pulse, leading to estimated generation densities up to $10^{19} \mathrm{~cm}^{-3}$ under band-to-band excitation. The pulse repetition rate was $10 \mathrm{~Hz}$. The time-resolving detection consisted of a single shot streak camera followed by a two-dimensional charge coupled device (CCD) array camera. It allowed a maximum resolution down to $5 \mathrm{ps}$, used to determine the temporal shape of the excitation pulse. Temporal nonlinearities as well as spatial and spectral dependences of the sensitivity of the detection systems were corrected by calibration algorithms. The pump-spot diameters varied around $100 \ldots 200 \mu \mathrm{m}$.

The luminescence collection was arranged in a backscattering geometry in all experimental setups to avoid reabsorption. For the measurements of the $\mathrm{CdS}_{0.94} \mathrm{Se}_{0.06}$ platelet sample the polarization directions of the excitation and the luminescence light were carefully adjusted to be perpendicular to the crystallographic $\vec{c}$-axis. All measurements were carried out at low temperatures between 5 and $8 \mathrm{~K}$, using liquid-helium cryostats.

\section{B. Steady-state luminescence}

\section{Luminescence of $\mathrm{ZnSe}_{1-c} \mathrm{Te}_{c}$}

In Fig. 4 are shown the calculated luminescence band of $\mathrm{ZnSe}_{1-c} \mathrm{Te}_{c}$ at $c=0.13$ and an experimental spectrum obtained under steady-state conditions at a low-density excitation. The mobility edge position equal to $0.18 \mathrm{eV}$ leads to satisfactory coincidence with the observed shift of the luminescence band with respect to the absorption maximum. ${ }^{4}$ The value of $\mathcal{P}\left(\omega_{M E}\right)$ was taken to be 1.4 , which corresponds to the result of Ref. 20 for the critical concentration of overlapping spheres. The comparison of the zero-phonon absorption (curve 1 in Fig. 4) and luminescence (curve 4) bands shows that only a small fraction of the tail states situated in the region of small values of the absorption coefficient and of the low density of the tail states is responsible for the steadystate luminescence band. The maximum of the zero-phonon luminescence band is considerably shifted with respect to the absorption band maximum. An additional Stokes shift of the luminescence band is produced in this case mainly by the interaction with LO phonons. The LO-phonon replicas are overlapping and form the broad luminescence band. In the calculations of the phonon-assisted processes the exciton wave function was taken in the form of Eq. (90) for the model II of localized excitons. Indeed, the localization ener-

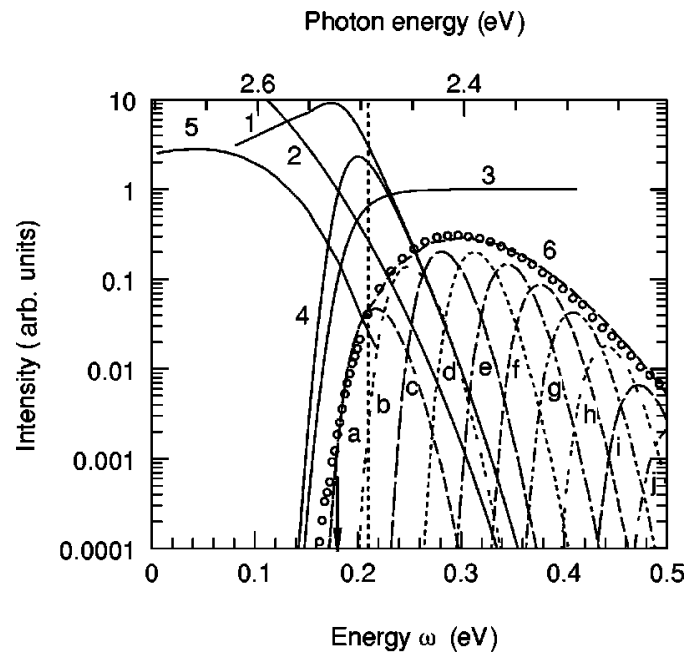

FIG. 4. Open circles-luminescence spectrum of $\mathrm{ZnSe}_{1-c} \mathrm{Te}_{c}$ at $c=0.13$ and $T=2 K$ (upper axis); full lines $(1-6)$ and broken lines $(a-j)$-calculated dependences: (1)_zero-phonon absorption band $\alpha_{1 s}^{0}(\omega),(2)$-normalized integral density of states $\mathcal{N}(\omega) / \mathcal{N}\left(\omega_{M E}\right)$, (3) -fraction of "radiative" states $P(\omega)$, (4) —zero-phonon luminescence band $I_{1 s}^{0}(\omega)$, (5)—absorption band $\alpha_{1 s}(\omega)$ and (6)luminescence band $I_{1 s}(\omega)$ via 1s-exciton state after taking into account exciton-phonon interaction; broken lines $(a-j)$-zero LOphonon luminescence band $(a)$ and its LO-phonon replicas of different orders ( $b-1 \mathrm{LO}, c-2 \mathrm{LO}$ etc.). Vertical arrow at $\omega=0.18 \mathrm{eV}$ indicates the estimated position of the mobility edge $\omega_{M E}$. Dotted vertical line corresponds to the excitation wavelength value used in luminescence decay experiments (see text).

gies $(\geqslant 0.1 \mathrm{eV})$ are considerably larger than the Coulomb binding energy of the exciton $(\approx 0.025 \mathrm{eV})$ for this system and electron is supposed not to be able to follow the hole motion adiabatically.

\section{Luminescence of $\mathrm{CdS}_{(1-c)} \mathrm{Se}_{c}$}

(1) Experimental data on the luminescence band shapes for $\mathrm{CdS}_{(1-c)} \mathrm{Se}_{c}$ for $c=0.20$ and $c=0.50$ and the comparison with calculated spectra are presented in Fig. 5.

All the parameters of the exciton-phonon interaction were obtained by means of linear interpolation of the values corresponding to the perfect crystals $\mathrm{CdS}$ and CdSe presented, for example, in Ref. 3. It was established that model I has to be used in the range of concentrations $c \geqslant 0.4$ to describe the exciton-phonon interaction. This region can be characterized by a relatively weak tailing where the binding energy of the exciton exceeds the localization energies and electron can follow adiabatically the motion of the hole over the potential well. Another situation takes place in the region $0.05<c$ $<0.4$, where the inequality $E_{e x}(c) \leqslant \omega_{M L}$ is realized between the exciton Coulomb energy $E_{e x}(c)$ and the typical localization energy of the tail states $\omega \approx \omega_{M L}$. Here the electron is not able to follow the hole motion adiabatically and the model II becomes more adequate. The transition from model I to model II is continuous and there exists a narrow interval of concentrations where the linear combination of the wave functions of the models I and II should be used. The effective strength of the exciton-phonon interaction changes significantly at the transition region from model I to model II because of the transformation of the wave function of the localized exciton. At the same time, the change of param- 

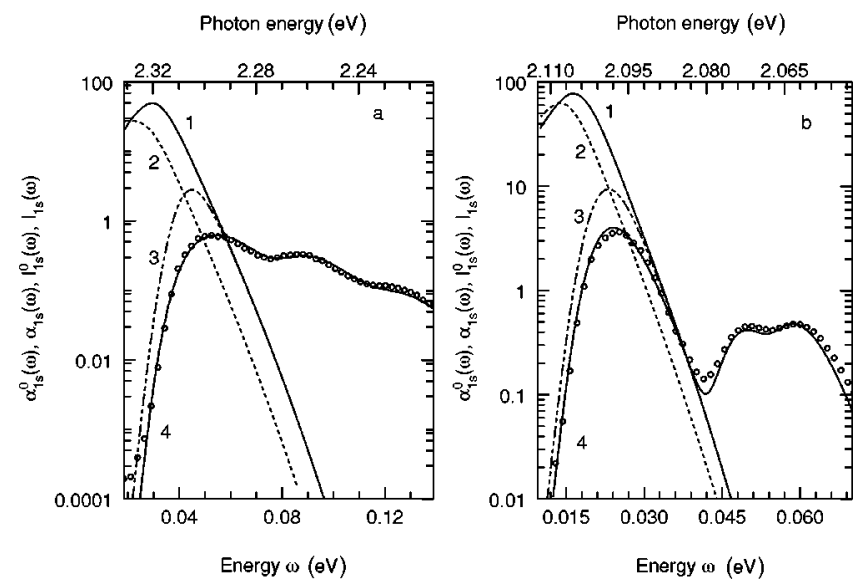

FIG. 5. Absorption and luminescence spectra of $\mathrm{CdS}_{1-c} \mathrm{Se}_{c}$ solid solution for $c=0.20(a)$ and $0.51(b)$ (upper energy axes). Open circles indicate the luminescence spectrum at band-to-band excitation. Lines give the result of computing for zero-phonon absorption band of the exciton ground state $\alpha_{1 s}^{0}(\omega)$ (1), absorption band taking into account interaction with phonons $\alpha_{1 s}(\omega)$ (2), zerophonon luminescence band $I_{1 s}^{0}(\omega)$ (3) and phonon-assisted luminescence band $I_{1 s}(\omega)(4)$.

eters of the Hamiltonian of the exciton-phonon interaction with composition of the solid solution gives only an insignificant effect and the common multiplication factor (of the order of unity), which was used in the fitting procedure, remains independent of composition for each type of interaction within the studied composition interval $0.05<c<0.6$. The change of the exciton-phonon interaction strength leads to considerably different shapes of the luminescence bands at different compositions of the solid solution. Fig. 5 demonstrates that the difference in the structure of the luminescence bands is most obvious in the region of the long wavelength wing of the spectra. In the composition interval where model I is applicable the exciton-phonon coupling is weak and the long wavelength wing of the main luminescence band reproduces the optical density of the tail states with high accuracy. In the region of model II the strength of the exciton-phonon interaction increases considerably causing modification of the long wavelength wing as compared to the zero-phonon band.

The slope of the short wavelength wing of the luminescence band is practically independent of the exciton-phonon interaction strength being a function of the composition. Both the Urbach parameter $\varepsilon_{U}(c)^{5}$ and the logarithmic slope of the short wavelength wing of the $\operatorname{band}^{8}\left(\left\{\ln \left[I_{1 s}^{0}(\omega)\right]\right\}_{\omega}^{\prime}\right)^{-1}$ reproduce the corresponding characteristics of the zerophonon luminescence band. The ratio of these characteristics remains constant with appropriate accuracy in a wide region of concentrations. ${ }^{8}$

(2) The luminescence spectrum of $\mathrm{CdS}_{(1-c)} \mathrm{Se}_{c}$ exhibits at relatively low concentration of Se $c<0.1$ new features as compared to previous data. The results of the comparison of the calculated and experimental luminescence bands of $\mathrm{CdS}_{(1-c)} \mathrm{Se}_{c}$ are given in Fig. 6 for $c \approx 0.05$. The observed band shows a well-pronounced structure, which results from LO-phonon replicas. There exists in this case a considerable deviation of the luminescence band shape calculated for the "radiative" states from the experimental one in the region of

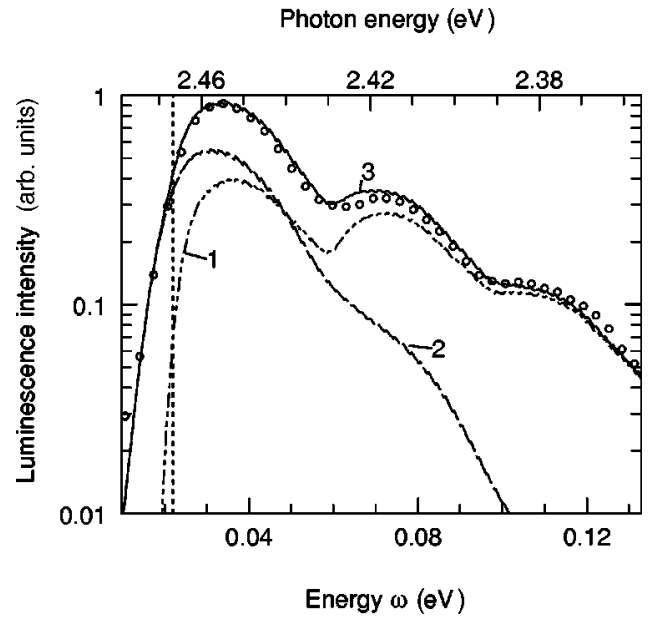

FIG. 6. Open circles show the luminescence spectrum of $\mathrm{CdS}_{1-c} \mathrm{Se}_{c}$ for $c=0.05$ at $T=2 \mathrm{~K}$ (upper energy axis). The lines are computed spectra of phonon-assisted recombination of "radiative" states (1), "silent" states (2) for $E_{F}=28 \mathrm{meV}$ and $T_{\text {eff }}$ $=2.75 \mathrm{meV}$ (see text) and sum of these two terms (3).

the short wavelength wing. To make the difference between experiment and theory more obvious we show the band calculated using the same approach as in the previous cases in Fig. 6 as curve 1. It is seen that the calculated band has a steeper slope of the short wavelength wing than the experimental luminescence band and the structure of the calculated LO-phonon replica also differs from the observed one.

To improve the agreement we assumed that in this case the condition of the low-excitation density is not fulfilled and "silent" states of the excitonic band have to be included into the consideration of the luminescence spectra. The following Fig. 7 exhibits the DOS and the integrated DOS data of "radiative" and "silent" states for this concentration, which illustrates the idea of the explanation. The integrated DOS of the "radiative" states has in this case with about $\approx 5.10^{16} \mathrm{~cm}^{-3}$ the lowest value among the considered solid solutions. If the intensity of excitation is not low enough then saturation of the "radiative" states will occur, which can be followed by the population of the "silent" states. If the number of populated "silent" states is comparable to the value of the integrated DOS of the "radiative" states then a considerable change of the luminescence band shape can take place. The other possible reason, which leads to the same effect, is slow rate of the exciton-phonon transitions from excited states of superclusters to their ground states because of too small an energy difference between these states in this composition region. At these conditions the "silent" states will be partially populated even when the saturation of the radiative states is not yet achieved.

The assumed additional emission of the "silent" states was described by Eq. (86) where the trial function $f(\omega)$ was used to fit the observed band shape. As a result, the fitting procedure shows that a relatively simple trial function $f(\omega)$ can be found if we exclude from the consideration the case of highest level of excitation and the shortest time delays in the other cases (see also Sec. III C and III D). With these exceptions the trial function $f(\omega)$ has the form of a quasiequilibrium Fermi distribution with two trial parameters, which is sufficient to describe the main features of the lumi- 


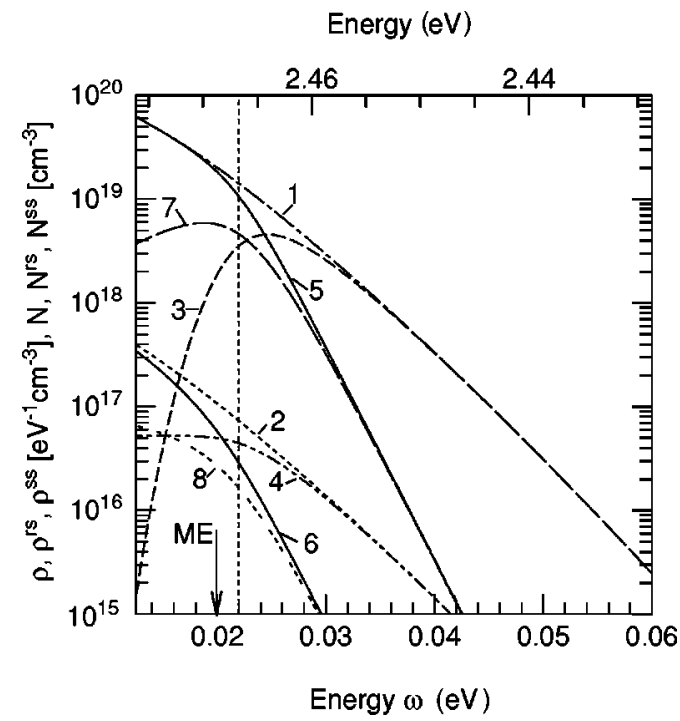

FIG. 7. Computed DOS's (odd numbers) and integrated DOS's (even numbers) curves of total $(1,2)$, "radiative" $(3,4)$, "silent" $(5,6)$, and populated "silent" states $(7,8)$ at $E_{F}=28 \mathrm{meV}$ and $T_{\text {eff }}=2.75 \mathrm{meV}$, respectively, for $\mathrm{CdS}_{1-c} \mathrm{Se}_{c}$ at $c=0.05$. The vertical arrow indicates the estimated position of the mobility edge $\omega_{M E}$. The dotted vertical line corresponds to the excitation wavelength value used in luminescence decay experiments for below band gap excitation (see text). The upper energy axes gives absolute energies for $\mathrm{CdS}_{1-c} \mathrm{Se}_{c}$ at $c=0.05$.

nescence band shape. In this case and below when we are considering modifications of the luminescence band shape at high excitation we take the function $f(\omega)$ in the form

$$
f(\omega)=\frac{1}{\exp \left[\left(\omega-E_{F}\right) / T_{e f f}\right]+1},
$$

where the trial parameters $E_{F}$ and $T_{e f f}$ were used to fit the shapes by means of the following equation

$$
I_{1 s}(\omega)=\int_{-\infty}^{0} d z I_{1 s}^{0}(\omega-z) F(z)+\int_{-\infty}^{0} d z \delta I_{1 s}^{0}(\omega-z) F(z) .
$$

Here $\delta I_{1 s}^{0}(\omega)$ is defined by Eq. (85) with the trial function $f(\omega)$ given by Eq. (94). The DOS and the integrated DOS of populated "silent" states obtained with the help of Eq. (94) are presented in Fig. 7 (curves 7 and 8). The additional emission band of "silent" states is presented in Fig. 6 as a curve 2 and the resulting band calculated as the sum of two bands 1 and 2 is given in Fig. 6 as curve 3 .

The fitting of the luminescence band shape shows that the effective exciton-phonon coupling strength for "silent" states is weaker than that for the "radiative" states. This result can be understood in view of different sizes of the states forming these two ensembles. The main part of the "radiative" states consist of isolated states and of the states belonging to pair superclusters while the "silent" states originate from the larger superslusters and from the percolation cluster. The size of the wave function directly influences the effective strength of exciton-phonon interaction through the restriction of the region of the Brillouin zone from which phonons participate in the emission process. We have found that the effective exciton-phonon coupling strength for "si- lent' states is to be taken approximately three times less than that for "radiative" states in order to fit the experimental spectrum.

\section{High-density excitation: modifications of the luminescence band shape}

High-density pulsed excitation gives a wide range of possibilities to study effects resulting from the saturation of the "radiative" states and the population of "silent" states. We discuss below the experimental data of two solid solutions, namely, $\mathrm{CdS}_{(1-c)} \mathrm{Se}_{c}$ at $c=0.05$ and $\mathrm{ZnSe}_{1-c} \mathrm{Te}_{c}$ at $c$ $=0.13$ which, are characterized by very different extents of their tail states. The first solid solution has about $10^{17} \mathrm{~cm}^{-3}$ states below the mobility edge, while the second has almost $10^{19} \mathrm{~cm}^{-3}$. Despite this difference, the behavior of the luminescence bands at high excitations are fairly similar. At excitation levels $0.3-1 \mathrm{~mJ} / \mathrm{cm}^{2}$ spectra detected during and just after the excitation pulse (during less than $0.5 \mathrm{~ns}$ for our conditions) show the features of the stimulated emission. These data and their discussion can be found in Refs. 24 and 26. Except for these shortest time delays and highest pumping intensities, the spectra and their modification with time delay are practically independent of the excitation power within the studied range $0.001-0.1 \mathrm{~mJ} / \mathrm{cm}^{2}$. This finding means that the concentration of excitons created in samples is restricted by bleaching of absorption at high level of excitation.

The result of model simulations and the experimental data for the evolution of the luminescence bands with time are shown in Fig. 8 for $\mathrm{CdS}_{(1-c)} \mathrm{Se}_{c}$ for $c=0.05$. Analogous data are given in Fig. 9 for $\mathrm{ZnSe}_{1-c} \mathrm{Te}_{c}$ for $c=0.13$. In both cases Eqs. (86), (94), and (95) were used in the calculations. The variable parameters of the fitting procedure $E_{F}$ and $T_{\text {eff }}$ of the trial function $f(\omega)$ are given in the captions of Figs. 8 and 9.

The constants of the exciton-phonon interaction were in both cases again decreased by a factor of 3 for the "silent" states as compared to the "radiative" states. The last fact can again be interpreted as the consequence of increase of an averaged size of wave functions of the "silent" states, which are formed by the large superclusters and by the percolation cluster.

\section{Low-density excitation: modifications of the luminescence band shape}

At Ti:Sa-laser $p s$-pulse excitation the luminescence spectra are in the first moment considerably less shifted into the blue region than at sub- $n s$ excitation discussed in the previous section. This finding confirms low-density conditions of the excitation.

In Figs. 10 and 11 the emission spectra at resonant excitation below $\omega_{M E}$ are shown for $\mathrm{CdS}_{(1-c)} \mathrm{Se}_{c}$ for $c=0.05$ and for $\mathrm{ZnSe}_{1-c} \mathrm{Te}_{c}$ for $c=0.13$. The spectra were integrated over different intervals of time. Figure 12 presents the luminescence spectra of $\mathrm{CdS}_{(1-c)} \mathrm{Se}_{c}$ for $c=0.05$ at band-toband excitation detected at similar conditions.

The spectra of $\mathrm{CdS}_{(1-c)} \mathrm{Se}_{c}$ show clearly the features of the exciton-phonon luminescence at all time delays. The shift toward the low energy increases with time delay and the 


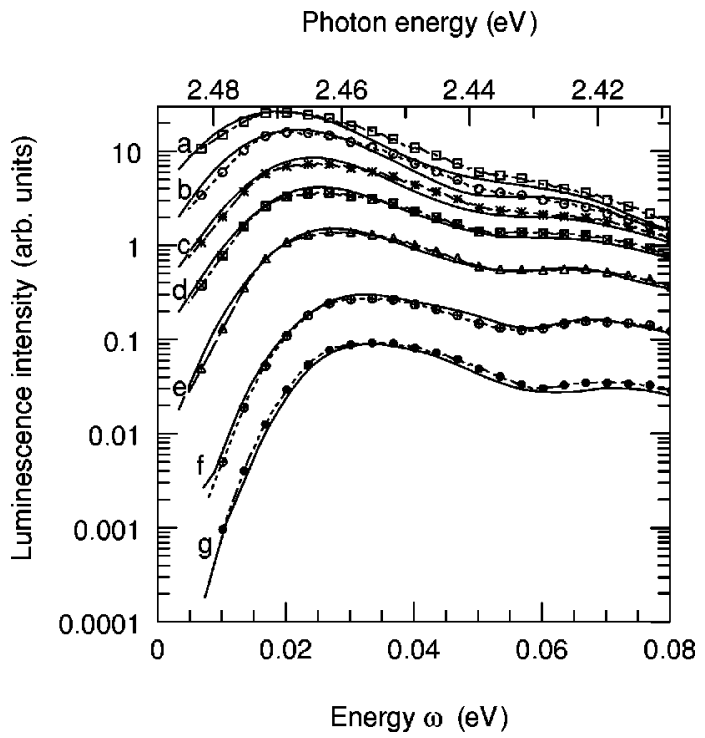

FIG. 8. Solid lines: luminescence spectra of $\mathrm{CdS}_{1-c} \mathrm{Se}_{c}, c$ $=0.05$ at $T=5 \mathrm{~K}$ for pulse ( $a-f$ curves) band-to-band excitation and different time delay after excitation pulse: $a-0.2, b-0.7, c$ $-1.5, d-2.5, e-4.5$, and $f-10 \mathrm{~ns}$, respectively. The luminescence spectrum at steady-state band-to-band excitation is shown by the solid curve $g$ (absolute energy values are shown at upper axis). Curves with symbols represent computed spectra of phononassisted recombination of "radiative" and "silent" states with trial function defined by Eq. (94) and the following fitting parameters $E_{F}$ and $T_{\text {eff }}$ (in meV): $a-0$ and $12 ; b-15$ and $7.5 ; c-25$ and 7.5; $d-25$ and $6.25 ; e-25$ and $4.25 ; f-28$ and 3 ; and $g-28$ and 2.75 , respectively.

change of the relation between zero-LO-phonon band and 1LO-phonon replica have the same character as it was discussed in Sec. III C.

\section{E. Pulse excitation: long-time kinetics of the luminescence}

The experimental data on spectrally integrated kinetics show that the luminescence process, which is characterized in a few first nanoseconds by a fast decay of the intensity exhibits afterwards a slow nonexponential behavior.

We consider as a reason for the long-time nonexponential kinetics the formation of excitons from separated electronhole pairs, which themselves are generated in the process of energy relaxation of electronic excitations produced by photons. In solid solutions with anion substitution the holes are more strongly affected by the fluctuation potential than the electrons and some part of photogenerated holes can be localized before the exciton formation occurs resulting in separated pairs even at excitation below the mobility edge.

The random potential influences the motion of electrons as well and leads to conduction band tailing. ${ }^{94}$ The formation of excitons is possible through the diffusion of mobile electrons or via tunneling of electrons, which were localized by the fluctuation potential of conduction band.

In this section we present the quantitative data on the time evolution of the luminescence during the long-time nonexponential stage of relaxation. The time behavior of the luminescence intensity during this stage depends on the energy of exciting photon and on the pulse power.

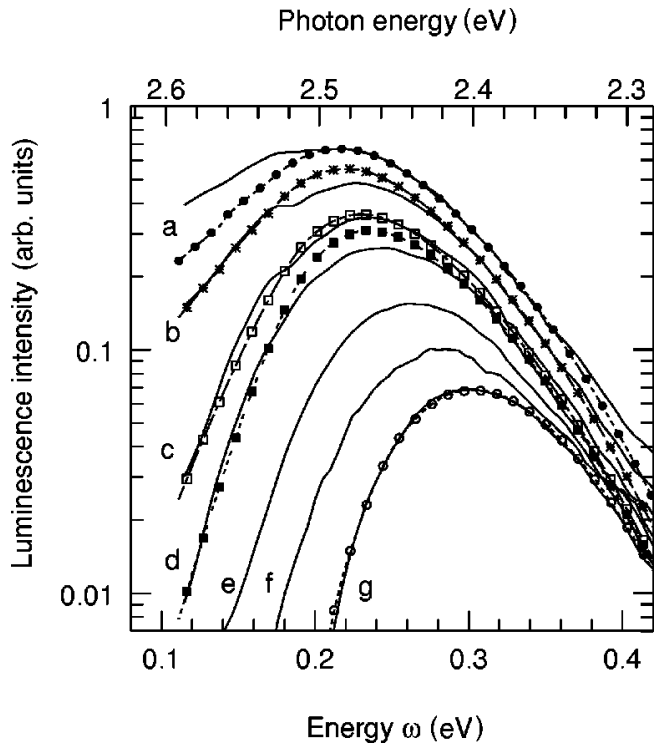

FIG. 9. Solid lines: luminescence spectra of $\mathrm{ZnSe}_{1-c} \mathrm{Te}_{c}, c$ $=0.13$ at $T=5 \mathrm{~K}$ for pulse ( $a-f$ curves) band-to-band excitation and different time delay after excitation pulse: $a-0.75, b-1.05$, $c-1.4, d-2.05, e-5.0$, and $f-50 \mathrm{~ns}$, respectively. The luminescence spectrum at steady-state band-to-band excitation is shown by solid curve $g$ (absolute energy values are shown at upper axis). Curves with symbols $a-d$ represent computed spectra of phononassisted recombination of "radiative" and "silent" states with the trial function defined by Eq. (94) and the following fitting parameters $E_{F}$ and $T_{\text {eff }}$ (in $\mathrm{eV}$ ): $a-0.05$ and $0.5 ; b-0.1$ and $0.1 ; c$ -0.14 and 0.035 ; and $d-0.15$ and 0.025 , respectively. Computed luminescence spectrum via the "radiative" states only is shown by broken curve $g$.

\section{CdS-Se long-time luminescence kinetics}

Figure 13 demonstrates the spectrally integrated kinetics of the luminescence in the long-time limit for the

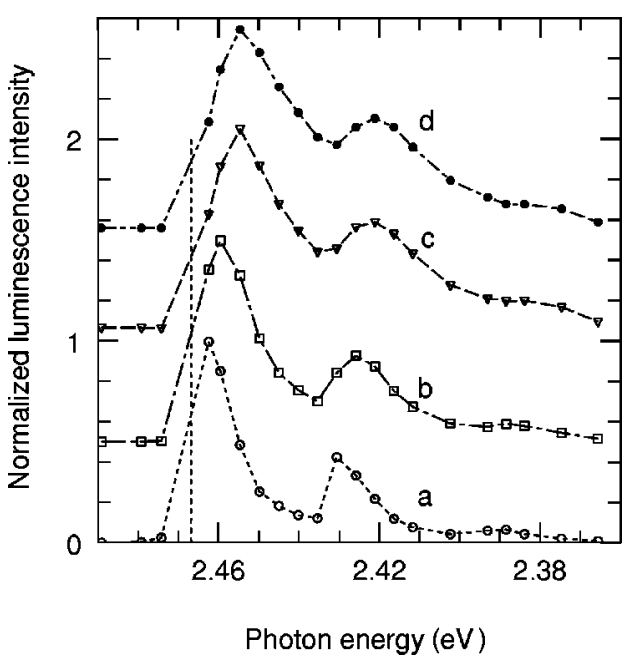

FIG. 10. Luminescence spectra of $\mathrm{CdS}_{1-c} \mathrm{Se}_{c}, c=0.05$ at resonant low-density pulse excitation at $2.467 \mathrm{eV}$ (dotted vertical line) integrated over the first nanosecond (curve $a$ ), and within the following time intervals after the excitation pulse: $1-5 \mathrm{~ns}, 5-50 \mathrm{~ns}$, and 50-450 ns (curves $b, c$, and $d$, respectively). All the spectra are shifted arbitrary on the ordinate for clarity. 


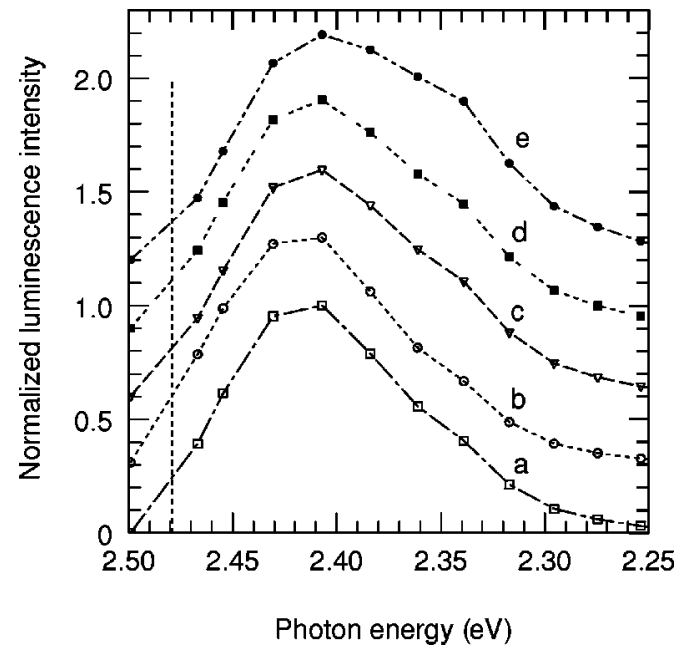

FIG. 11. Luminescence spectra of $\mathrm{ZnSe}_{1-c} \mathrm{te}_{c}, c=0.13$ at resonant low-density pulse excitation at $2.479 \mathrm{eV}$ integrated over the first nanosecond (curve $a$ ), and within the following time intervals after the excitation pulse: $1-5 \mathrm{~ns}, 5-50 \mathrm{~ns}$, and 50-450 ns (curves $b, c$, and $d$, respectively). All the spectra are shifted arbitrary on the ordinate. The excitation wavelength position is indicated by a vertical dotted line.

$\mathrm{CdS}_{(1-c)} \mathrm{Se}_{c}$ sample with $c=0.05$ at a low density of excitation in the spectral region below the exciton mobility edge (curve 1).

The experimentally observed intensity decrease in this case can be fitted by the power law

$$
I(t) \sim[(1+t / \tau)]^{-\delta},
$$

with $\delta=2$. This type of behavior is usually attributed to bimolecular annihilation reactions, which are described in the mean-field approximation by the equation

$$
\frac{d n_{e}}{d t}=\frac{d n_{h}}{d t}=-k n_{e} n_{h},
$$

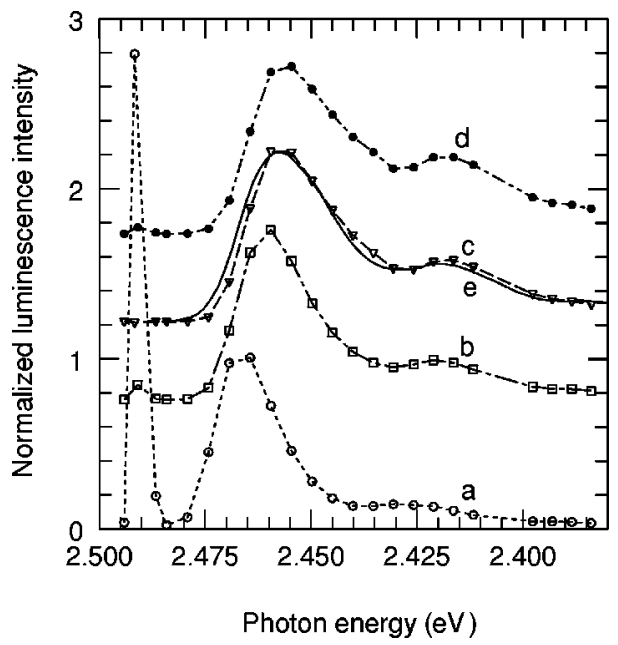

FIG. 12. Luminescence spectra of $\mathrm{CdS}_{1-c} \mathrm{Se}_{c}, c=0.05$ at the low-density pulse excitation at $2.49 \mathrm{eV}$ integrated over the first nanosecond (curve $a$ ), and within the following time intervals after excitation pulse: $1-5 \mathrm{~ns}, 5-50 \mathrm{~ns}$, and $50-450 \mathrm{~ns}$ (curves $b, c$, and $d$, respectively). The luminescence spectrum at steady-state band-to-band excitation is also indicated by the solid line $e$.

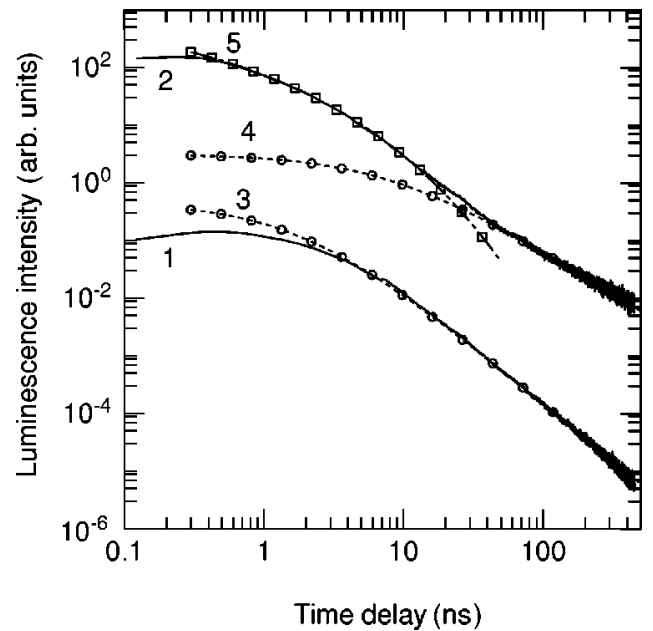

FIG. 13. Decay of integrated luminescence spectra of $\mathrm{CdS}_{1-c} \mathrm{Se}_{c}, c=0.05$ at low-density excitations below and above the mobility edge (solid lines 1 and 2, respectively). Excitation wavelengths correspond to $\omega=0$ and $\omega=0.022 \mathrm{eV}$ in Fig. 6 and Fig. 7. Open circles 3 and 4 represent the fit of the long-time kinetics by power the law of Eq. (96) with $\delta$ equal to 2 and 3/2, respectively, with parameter $\tau$ equal to 1.81 and $7.85 \mathrm{~ns}$. Open squares 5 indicate a stretched exponential dependence Eq. (104) with $\tau_{D}^{\prime}$ $=0.043 \mathrm{~ns}$ and $\delta=0.33$.

where $n_{e} \approx n_{h}$ are the averaged electron and hole concentrations and $k$ is the time independent averaged probability of recombination (or the reaction rate constant). The dependence given by this equation presents a particular case of the well-known Becquerel's law. ${ }^{40}$

The obtained results can be explained in the framework of the above proposed concept, which supposes that a fraction of the absorbed photons produces particles separated in the space. Under excitation by $2.467 \mathrm{eV}$ used in this experiment the immobile "radiative" states are up one half of the total number of states excited by photons (see Fig. 7). The remaining half of the states is supposed to be able to generate separated pairs during energy relaxation.

According to Refs. 41-43 we have to expect different asymptotic behavior for the temporal decrease of the luminescence in dependence on the character of the motion of the annihilating particles. The observed results agree well with the dependence for the localized particles for which the radiative recombination is possible only through tunneling, like in the case of donor-acceptor pairs. ${ }^{39}$

The mean-field approximation assumes a spatially random and homogeneous in space distribution of annihilating particles and neglects possible correlations of their distribution or fluctuations. The good correspondence of observed time dependence of the integrated luminescence intensity to the solution of Eq. (97) indicates the validity of the above assumption. The spectral position of maximum and the shape of the luminescence band coincide at long times practically with those of the steady-state luminescence. So, we can conclude that localized exciton formation is responsible for the long-time luminescence decay followed by its radiative recombination.

Figures 13 (curve 2) and 14 present the time kinetics of the same sample of $\mathrm{CdS}_{(1-c)} \mathrm{Se}_{c}$ under the excitation above the mobility edge at low and high excitation, correspond- 


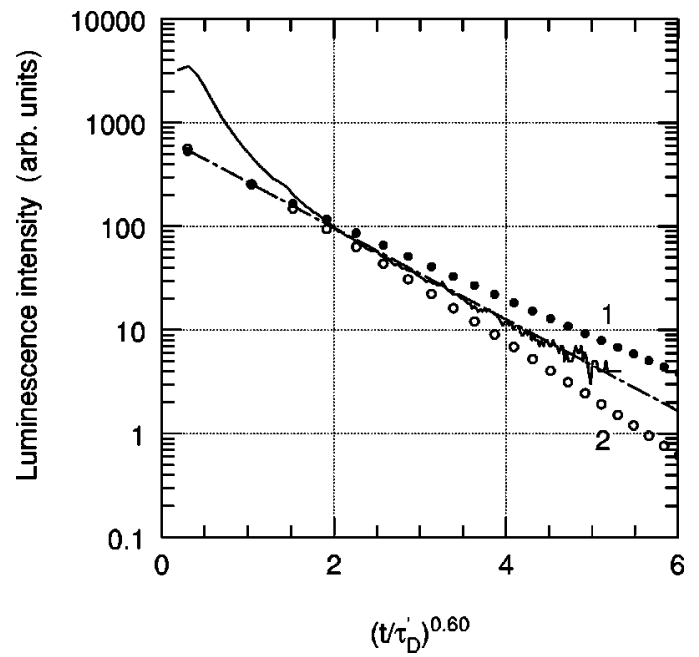

FIG. 14. Decay of integrated luminescence spectra of $\mathrm{CdS}_{1-c} \mathrm{Se}_{c}, c=0.05$ at high-density excitation above the mobility edge with density $0.2 \cdot 10^{-3} \mathrm{~J} / \mathrm{cm}^{2}$ (solid line) in a semilogarithmic plot as a function of $t^{0.6}$. The dashed line represents a stretched exponential fit by Eq. (104) with $\tau_{D}^{\prime}=1.0 \mathrm{~ns}$ and $\delta=0.60$. Symbols 1 and 2 represent the fit with $\delta=0.55$ and 0.65 , respectively.

ingly. The behavior of the intensity differs considerably from that in the previous case. Namely, the decay of the $\mathrm{CdS}_{(1-c)} \mathrm{Se}_{c}$ luminescence obeys in an intermediate time interval at low excitation the stretche-exponential law $\exp \left(-t^{\delta}\right)$ with the index $\delta \approx 0.33$, while this dependence transforms into a power $t^{-3 / 2}$ law in the limit of long times, which means considerable decrease of the recombination rate. At high intensity of excitation the time kinetics of the same $\mathrm{CdS}_{(1-c)} \mathrm{Se}_{c}$ sample follows the stretched-exponential behavior with the critical index $\delta \approx 0.6$.

We will try to describe the results for these cases using the approach of the so-called diffusion controlled annihilation $^{41-43}$ where the time dependence of the particle concentration and of the reaction rate is strongly inflicted by the fluctuations of the particle concentration.

Let us suppose that the particles of both kind are mobile and the averaged concentration of them are equal. Then the random walk of both particles leads to their collision followed by the exciton formation and its annihilation during radiative lifetime. If the time of exciton formation and its radiative time are less than the time of diffusion over the microscopic volume $L^{d}$

$$
\tau_{D} \sim L^{2} / D,
$$

then the number of particles in this volume (see Refs. 41 and 43) decreases because of annihilation during $\tau_{D}$ from its initial value

$$
n_{e, h}(0) L^{d} \pm\left[n(0) L^{d}\right]^{1 / 2}
$$

to the value

$$
n_{e, h}\left(\tau_{D}\right) \sim\left[n_{e, h}(0) L^{d}\right]^{1 / 2} / L^{d} \sim L^{-d / 2} \sim \tau^{-d / 4} .
$$

This leads to the time dependence of the recombination intensity of the form

$$
I(t) \sim d n_{e, h} / d t \sim t^{-(4+d) / 4} .
$$

Here $D$ is the diffusion coefficient for the most mobile particles and $d$ is the dimensionality of the space.

This kind of dependence was established in the theory by different methods (see, e.g., Refs. 42 and 49). However, the experimental data for $\mathrm{CdS}_{(1-c)} \mathrm{Se}_{c}$ cannot be fitted by this law in the case under consideration. This fact confirms the assuming that at least the particles of one type are localized in this system.

For the case of mobile electrons and localized holes the recombination should be considered as a result of a random walk of electrons in search of localized holes and the recombination kinetics should keep the peculiarities and consequences of this process. To describe the long-time behavior of the luminescence intensity we have to investigate the electron motion at times essentially exceeding the time of electron hops within the volume of size $L$, which is restricted by the localized holes. The time dependence for an electron concentration in this volume can be written as

$$
\frac{d n_{e}}{d t} \sim-\frac{n_{e}}{\tau_{D}},
$$

where $\tau_{D}$ is defined again by Eq. (98) with $D$ equal to the diffusion coefficient for electrons. If only a fraction of electrons is mobile and the number of mobile electrons is considerably less than the number of holes the simple exponential decay of the electron concentration follows also from Eq. (97).

Taking into account the Poisson fluctuations in the space distribution of holes we obtain for the probability of the realization of a volume $L^{d}$ empty of holes

$$
P\left(L^{d}\right)=\exp \left(-n_{h} L^{d}\right) .
$$

Averaging over the space distribution of holes gives the stretched-exponential dependence $e^{41-48}$

$$
\exp \left(-\left[t / \tau_{D}^{\prime}\right]^{\delta}\right),
$$

for both $n_{e}(t)$ and $I(t) \sim d n_{e} / d t$ with the critical index of "normal" diffusion $\delta=d /(d+2)$ where $\tau_{D}^{\prime}$ is the characteristic time of the diffusion process.

The main contribution to the long-time dynamics of the luminescence comes from the regions free of holes of "optimal" size $R_{\text {opt }}$ (Ref. 91)

$$
R_{o p t}(t) \sim\left(\frac{D t}{n_{h}}\right)^{(1 /(d+2))} .
$$

A more pronounced slowing down of the annihilation process occurs if the geometry of the percolation cluster restricts the trajectories of the moving particle. An important characteristic of the percolating system is the so-called correlation length $\xi$, which gives the averaged size of finite clusters or voids (holes) in the infinite cluster. ${ }^{22}$ When the optimal size $R_{o p t}$ has the order of the correlation length of the percolation cluster $R_{o p t} \leqslant \xi$ the diffusion process attains the 


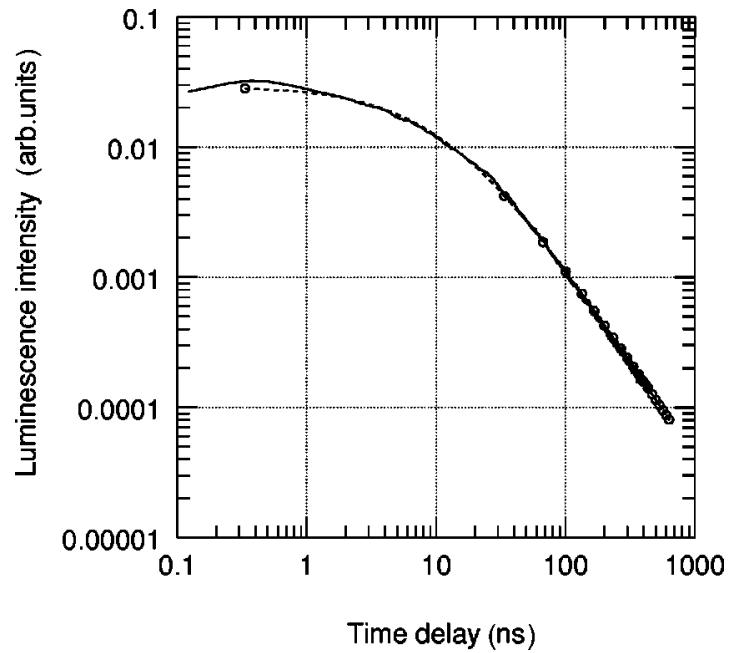

FIG. 15. Decay of integrated luminescence spectra of $\mathrm{ZnSe}_{1-c} \mathrm{Te}_{c}, c=0.13$ at low-density excitations below the mobility edge (solid line). Excitation wavelength corresponds to the dotted vertical line in Fig. 2. Dashed line with symbols represents the fit of the long-time kinetics by the power law of Eq. (96) with the parameters $\tau=14.1$ ns and $\delta=3 / 2$.

"anomalous" 41,43 character with a substantial decrease of the diffusion rate. In the case of diffusion over the percolation cluster we have $\mathrm{e}^{90,91}$

$$
\ln [I(t)] \sim-t^{\left[d_{s} /\left(d_{s}+2\right)\right]},
$$

where $d_{s}=2 d_{f} / d_{w}$ is the "hyper-universal" fraction dimensionality, ${ }^{92,93}$ i.e., the spectral dimensionality of percolation cluster. Here $d_{f}$ is the fractal dimensionality $\left(d_{f}\right.$ $\approx 2.51$ for $d=3)^{22}$, and $d_{w}$ is the index of random walk over the fractal $\left(d_{w} \approx 3.8\right.$ for $\left.d=3\right) .^{43}$

A further decrease of the diffusion rate occurs when the diffusion takes place partly on clusters of finite size. This leads to a change of $d_{s}$ in Eq. (106)

$$
d_{s} \rightarrow d_{s}^{\prime}=2 d_{f} / d_{w}^{\prime},
$$

where $d_{w}^{\prime}=d_{w} /(1-\beta / 2 \nu)^{43}[\beta$ and $\nu$ are the critical indexes of the order parameter and correlation length, for $d=3, \beta$ $\approx 0.42, \nu \approx 0.875$, and $d_{w}^{\prime} \approx 5.0$ (Ref. 22)].

The observed difference of the stretched-exponential indexes of the data presented in Figs. 13 and 14 might be assigned to the different filling of the extended electron states at different pumping levels. The higher intensity of excitation leads to a higher concentration of electrons and holes. The ratio of the correlation length of the electron percolation cluster $\xi$ to the optimal diffusion length $R_{o p t}$ depends on the carrier concentration $n_{h} \approx n_{e}$ and defines the character of the diffusion process, whether it is normal or anomalous. ${ }^{41,43}$ Though both functions are decreasing with concentrations, the rate of the decrease of $\xi \sim\left(n_{e}-n_{e}^{\text {crit }}\right)^{-\nu}$ exceeds that of $R_{\text {opt }}$ given by Eq. (105) and, therefore, their ratio $\xi / R_{o p t}$ tends toward values less than unity with increasing concentration, which characterizes the normal diffusion.

The data of Fig. 13 (curve 2) show that a stretchedexponential law $\exp \left(-t^{\delta}\right)$ with the index $\delta \approx 0.33$ transforms

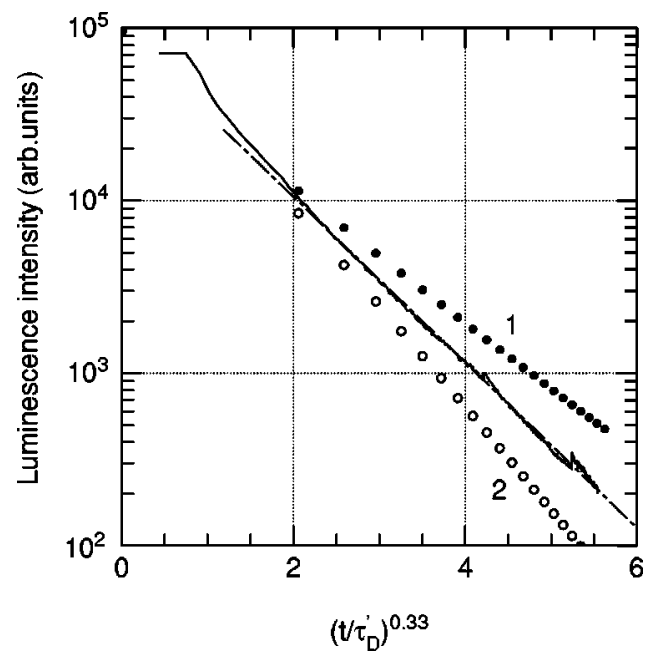

FIG. 16. Decay of integrated luminescence spectra of $\mathrm{ZnSe}_{1-c} \mathrm{Te}_{c}, c=0.13$ at high-density excitation above the mobility edge with density $10^{-3} \mathrm{~J} / \mathrm{cm}^{2}$ (solid line) in semilogarithmic plot as a function of $t^{0.33}$. Dashed line represents a stretched exponential fit by Eq. (104) with $\tau_{D}^{\prime}=0.74$ ns and $\delta=0.33$. Symbols 1 and 2 represent the fit with $\delta=0.30$ and 0.36 , respectively.

into a power $t^{-3 / 2}$ law in the limit of long times. This kind of dependence also can be connected with diffusion.

Noolandi et al. ${ }^{50}$ have proposed a model of geminate electron-hole pair recombination for $\alpha-\mathrm{Si}: \mathrm{H}$, involving tunneling and diffusion. Within the so-called prescribed diffusion approximation ${ }^{51}$ they have take into account the attractive Coulomb interaction between electron and hole. According to their results, the intensity should decrease in the long-time limit as $t^{-3 / 2}$, which describes well the luminescence decay in amorphous silicon.

Kuzovkov and Kotomin ${ }^{49}$ have considered a complicated system of equations for the diffusion-controlled bimolecular reaction. The solution describes the development of an inhomogeneity in the space distribution of the particles leading to the time dependence of the bimolecular reaction rate $k$ $\rightarrow k(t)$. As a result, it has been found in the long-time limit that the reaction rate varies according to power law $k$ $\sim t^{-1 / 2}$ for the case of equal concentrations of both mobile and immobile particles. The particle concentration decreases in this case as $n_{e} \sim t^{-1 / 2}$. This leads to the decay of the luminescence intensity also according to power law $I(t)$ $\sim t^{-3 / 2}$. This result follows also from Eq. (97) if the bimolecular reaction rate $k$ has the mentioned power dependence on time. ${ }^{49}$

Thus, the long time dependence of the type $I(t) \sim t^{-3 / 2}$ follows from two different models of kinetics considered in Refs. 49 and 50. The simplest explanation of this fact can be as follows. According to Refs. 49 and 42 the decrease of concentration $n_{e} \sim t^{-1 / 2}$ indicates an inhomogeneous distribution of particles, which is developing in the process of diffusion. We assume that the inhomogeneous distribution of the remaining particles produced by the diffusion during the intermediate time interval leads to the decrease of annihilation rate as compared with the case of a random distribution also when the remaining electrons are localized and tunneling is responsible for their annihilation. 


\section{ZnSe-Te long-time luminescence kinetics}

Figures 15 and 16 exhibit data for $\mathrm{ZnSe}_{(1-c)} \mathrm{Te}_{c}$ solid solution with $c=0.13$ investigated at low excitation slightly below and at high excitation slightly above the mobility edge. The time dependence of the luminescence intensity shows the power $t^{-3 / 2}$ asymptotic behavior at low excitation and the stretched-exponential dependence with the index of anomalous diffusion $\delta \approx 0.33$ at high excitation over a wide time interval. The explanation of the previous case can be used here as well. Despite the fact that at low excitation the photon energy was below the mobility threshold the number of "nonradiative" states of superclusters of large size exceeds considerably the number of "radiative" states in the spectral region of excitation (see Fig. 4). As a consequence, the processes of the fast relaxation of holes, which electrons cannot follow adiabatically, creates favorable conditions for the generation of the separated pairs. The scale of the random potential for electrons in $\mathrm{ZnSe}_{(1-c)} \mathrm{Te}_{c}$ is supposed to exceed considerably that in $\mathrm{CdS}_{(1-c)} \mathrm{Se}_{c}$ and we can admit that the electron concentration achieved in the former case is not sufficiently high to break the relation between the correlation length and the optimal diffusion length, which should lead to anomalous diffusion. The results at weak excitation show in this case the $t^{-3 / 2}$ decrease (which, probably, should be considered as the most common law in the long-time limit) while this limit was not achieved in our experiments even at highest excitations.

\section{F. Some further remarks}

The nonexponential stage of relaxation lasts tens and even hundreds of nanoseconds and the observed time dependences can be understood under the assumption that the relaxation rate is controlled by diffusion of separated particles or by their tunnelling. This stage of the process looks like separated nongeminate pair recombination. In the whole, only a small fraction of excitations participates in these processes.

One of the most important questions is whether this longtime kinetics is an intrinsic or extrinsic property of radiative recombination of the solid solutions. We have found very similar luminescence kinetics in the CdS-Se samples grown by hotwall epitaxy and in high-quality samples obtained by high-temperature growth from gaseous phase. Similar situation has been found for time kinetics of the luminescence of two different $\mathrm{ZnSe}_{(1-c)} \mathrm{Te}_{c}$ samples with $c=0.11$ and $c$ $=0.13$ obtained at different conditions. Since the ensembles of point defects, the dislocation density, as well as the impurity contents are different in this set of the samples, the natural conclusion would be that the observed features of the long decay kinetics are of an intrinsic nature.

It is worth mentioning that even the short review of some of the possible consequences of the tunneling and diffusion in disordered systems shows that these processes could account for quite different types of luminescence intensity dependences at the condition that some fraction of excited states occurs in the form of separated electron-hole pairs. The explanation of the results presented here is based on the idea that the motion of separated particles, both electrons and holes, is affected by the random potential of solid solutions leading to tailing of both valence and conduction bands. Then the diffusion of mobile carriers or tunneling of local- ized electrons followed by exciton formation and their radiative recombination could explain the long-time survival of the excitation.

Another serious question is the problem of uniqueness of the presented interpretation. The problem arises, for instance, since it is difficult to distinguish Becquerel's law with $\delta$ $=2$ from stretched-exponential decrease with $\delta=0.33$ within a relatively narrow interval of observation in our measurements of $\mathrm{CdS}_{(1-c)} \mathrm{Se}_{c}$ (see Fig. 13). We have preferred to fit by stretched-exponential functions in all appropriate cases because they describe the decrease of intensity over the widest interval of time we were dealing with and give the possibility to include without contradiction both normal and anomalous diffusion.

The other example of some uncertainty presents the stretched-exponential dependence itself. Presently such a dependence can be strictly justified only if considerable concentration differences of mobile particles and traps exist. Therefore, additional study is necessary to establish whether or not it can be proved for electrons the concentration of which coincides with that of holes, but with electrons are distributed over extended and localized states in the disordered system.

In any case, the experimental data show that the stretchedexponential decrease can serve only as an intermediate time asymptotics, while the long-time behavior is consistent with a $t^{-3 / 2}$ law. According to Refs. 49 and 50, this latter dependence can be the consequence either of the electron-hole Coulomb attraction or of the spatially inhomogeneous distribution of particles, which is developing in the preceding process of diffusion. In the former case it is difficult to explain the decay behavior at intermediate times. In the latter case we have to suppose that approximately the same $t^{-3 / 2}$ dependence takes place also for the tunneling processes of inhomogeneously distributed electrons.

\section{CONCLUSIONS AND OUTLOOK}

Semiconductor alloys of the type $A B_{1-{ }_{c}} C_{c}$ form a set of disordered solids properties of which vary in wide limits. The experience accumulated already in the investigation of these objects and the growing interest to their technological applications make it useful to attempt to highlight some common features of the solid solutions, which are different in many details.

In this paper we have presented the model based on the classical percolation theory for the description of the luminescence process. This approach exploiting the fractal geometry of percolation cluster turns out to be fruitful for the description of the steady-state luminescence under weak stationary conditions of very different systems.

The population dynamics after pulsed excitation gives new features to the luminescence. We have presented the description of this process in terms of the approach developed for stationary conditions. The deviations of experimental data from the theory have in this case a predictable character. They are caused, first of all, by the fact that the theory dealing with the geometrical figures does not take into account transformations of wave functions of the tail states in the mobility edge region.

A nontrivial long-time kinetics of the luminescence, 
which has been observed, touches upon the problem of the separated pair generation under excitation even below the mobility edge of excitons. We have shown that the observed long-time decay of the spectrally integrated intensity of the luminescence can be interpreted in terms of theories of bimolecular reactions and open questions for further study are partly presented in Sec. III F.

Apart from a detailed understanding of the tail states in three-dimensional solids, the knowledge obtained here seems to be of great help to understand analogous phenomena in systems of reduced dimensionality.

\section{ACKNOWLEDGMENTS}

The paper presented here is the result of the cooperation of several research groups in different countries. The authors would, therefore, like to express their thanks to funding agencies supporting the scientific work and/or the exchange of scientists. Special thanks are due to the Deutsche Forschungsgemeinschaft, the European Community in the frame of the INTAS program (Grant No. 94-324), and the Russian Foundation for Basic Research (Grants Nos. 96-02-16933 and 97-02-18138).
${ }^{1}$ R.A. Street, Adv. Phys. 30, 593 (1981).

${ }^{2}$ S. Permogorov, A. Reznitsky, V. Travnikov, S. Verbin, G.O. Müller, P. Flögel, and M. Nikiforova, Phys. Status Solidi B 113, 589 (1982)

${ }^{3}$ E. Cohen and M. Sturge, Phys. Rev. B 25, 3828 (1982).

${ }^{4}$ S. Permogorov and A. Reznitsky, J. Lumin. 52, 201 (1992).

${ }^{5}$ A. Naumov, S. Permogorov, A. Reznitsky, S. Verbin, and A. Klochikhin, J. Cryst. Growth 101, 713 (1990).

${ }^{6}$ A.G. Abdukadyrov, S.D. Baranovskii, S.Yu. Verbin, E.L. Ivchenko, A.Yu. Naumov, and A. Reznitsky, Zh. Eksp. Teor. Fiz. 98, 2056 (1990) [Sov. Phys. JETP 71, 1155 (1990)].

${ }^{7}$ E.L. Ivchenko and A. Reznitsky, Philos. Mag. B 65, 733 (1992).

${ }^{8}$ A. Reznitsky, S.D. Baranovskii, A. Tsekoun, and C. Klingshirn, Phys. Status Solidi B 184, 159 (1994).

${ }^{9}$ A. Klochikhin, S. Permogorov, and A. Reznitsky, J. Cryst. Growth 159, 848 (1996).

${ }^{10}$ A.A. Klochikhin, S.A. Permogorov, and A.N. Reznitsky, Pis'ma Zh. Éksp. Teor. Fiz. 65, 274 (1997) [JETP Lett. 65, 289 (1997)].

${ }^{11}$ A.A. Klochikhin, S.A. Permogorov, and A.N. Reznitsky, Phys. Solid State 39, 1035 (1997).

${ }^{12}$ A.A. Klochikhin, S.A. Permogorov, and A.N. Reznitsky Phys. Solid State 40, 1290 (1998).

${ }^{13}$ M. Queslati, C. Benoit á la Guillaume, and M. Zouaghi, Phys. Rev. B 37, 3037 (1988).

${ }^{14}$ A.A. Klochikhin and S.G. Ogloblin, Phys. Rev. B 48, 3100 (1993).

${ }^{15}$ A.A. Klochikhin, Phys. Rev. B 52, 10979 (1995).

${ }^{16}$ V.K.S. Shante and S. Kirkpatric, Adv. Phys. 20, 325 (1971).

${ }^{17}$ J.W. Essam, Rep. Prog. Phys. 43, 833 (1980).

${ }^{18}$ D.F. Holcomb and J.J. Rehr, Jr., Phys. Rev. 183, 733 (1969).

${ }^{19}$ G.E. Pike and C.H. Seager, Phys. Rev. B 10, 1421 (1974).

${ }^{20}$ S.W. Haan and R. Zwanzig, J. Phys. A 10, 1547 (1977).

${ }^{21}$ W.T. Elam, A.R. Kerstein, and J.J. Rehr, Jr., Phys. Rev. Lett. 52, 1516 (1984).

${ }^{22}$ A. Bunde and S. Havlin, in Fractals and Disordered Systems, edited by A. Bunde and S. Havlin (Springer-Verlag, Berlin, 1994), p. 51.

${ }^{23}$ H. Haug and S.W. Koch, Quantum Theory of the Optical and Electronic Properties of Semiconductors (World Scientific, Singapore, 1994).

${ }^{24}$ T. Breitkopf, A. Reznitsky, H. Kalt, and C. Klingshirn, J. Cryst. Growth 159, 788 (1996).

${ }^{25}$ M. Grün, H. Gerlach, T. Breitkopf, M. Hetterich, A. Reznitsky, H. Kalt, and C. Klingshirn, J. Cryst. Growth 146, 414 (1994).

${ }^{26}$ T. Breitkopf, H. Kalt, C. Klingshirn, and A. Reznitsky, J. Opt. Soc. Am. B 13, 1251 (1996).
${ }^{27}$ R. Westphäling, T. Breitkopf, S. Bauer, and C. Klingshirn, J. Lumin. 72-74, 980 (1997).

${ }^{28}$ H. Schwab, C. Dörnfeld, E.O. Göbel, J.M. Hvam, C. Klingshirn, J. Kuhl, V.G. Lyssenko, F.A. Majumder, G. Noll, J. Nunnenkamp, K.H. Pantke, R. Renner, A. Reznitsky, U. Siegner, H.E. Svoboda, and Ch. Weber, Phys. Status Solidi B 172, 479 (1992).

${ }^{29}$ C. Klingshirn, Phys. Status Solidi B 202, 857 (1997).

${ }^{30}$ F.A. Majumder, S. Shevel, V.G. Lyssenko, H.E. Svoboda, and C. Klingshirn, Z. Phys. B 66, 409 (1987).

${ }^{31}$ U. Siegner, D. Weber, E.O. Göbel, D. Bennhardt, V. Heuckeroth, R. Saleh, S.D. Baranovskii, P. Thomas, H. Schwab, C. Klingshirn, J.M. Hvam, and V.G. Lyssenko, Phys. Rev. B 46, 4564 (1992).

${ }^{32}$ S. Permogorov, A. Reznitsky, S. Verbin, and V. Lyssenko, Solid State Commun. 47, 5 (1983).

${ }^{33}$ H. Schwab, V. Lyssenko, A. Reznitsky, and C. Klingshirn, J. Lumin. 48-49, 661 (1991).

${ }^{34}$ A.N. Reznitsky, A.V. Kornievsky, A.A. Kiselev, S. Permogorov, L.N. Tenishev, A.A. Klochikhin, S. Verbin, H. Gerlach, M. Hetterich, M. Grün, and C. Klingshirn, Phys. Solid State 40, 829 (1998).

${ }^{35}$ J.A. Kash, A. Ron, and E. Cohen, Phys. Rev. B 28, 6147 (1983).

${ }^{36}$ V. Agekyan, R. Bindemann, R. Schwabe, Y. Stepanov, and I. Streit, Phys. Status Solidi B 116, K43 (1983).

${ }^{37}$ Ja. Aaviksoo, Ja. Lippmaa, S. Permogorov, A. Reznitsky, P. Lavallard, and K. Gourdon, Pis'ma Zh. Éksp.Teor. Fiz. 45, 391 (1987) [JETP Lett. 45, 498 (1987)].

${ }^{38}$ S. Permogorov, A. Reznitsky, J. Aaviksoo, J. Lippmaa, K.Gourdon, and P. Lavallard, in Ultrafast Phenomena in Spectroscopy, edited by Z. Rudzikas, A. Piskarskas, and R. Baltramejunas (World Scientific, Singapore, 1988), p. 473.

${ }^{39}$ D.G. Thomas, J.J. Hopfield, and W.M. Augustyniak, Phys. Rev. 140, A202 (1965).

${ }^{40} \mathrm{E}$. Becquerel, La Lumière, ses causes et ses effects (Firmin Didot, frères, fils et cie, Paris, 1868).

${ }^{41}$ S. Havlin and D. Ben-Avraham, Adv. Phys. 36, 695 (1987).

${ }^{42}$ V. Kuzovkov, and E. Kotomin, Rep. Prog. Phys. 51, 1479 (1988).

${ }^{43} \mathrm{~S}$. Havlin and A. Bunde, in Fractals and Disordered Systems, edited by A. Bunde and S. Havlin (Springer-Verlag, Berlin, 1994), p. 97.

${ }^{44}$ B. Ja. Balagurov and V.G. Vax, Zh. Éksp. Teor. Fiz. 65, 1939 (1973) [Sov. Phys. JETP 38, 968 (1974).

${ }^{45}$ A.A. Ovchinnikov and Ya. B. Zeldovich, Chem. Phys. 28, 215 (1978).

${ }^{46}$ P. Meakin and H.E. Stanley, J. Phys. A 17, L173 (1984).

${ }^{47}$ K. Kang and S. Redner, Phys. Rev. Lett. 52, 955 (1984). 
${ }^{48}$ N.D. Donsker and S.R.S. Varadhan, Commun. Pure Appl. Math. 32, 721 (1979).

${ }^{49}$ V.N. Kuzovkov and E.A. Kotomin, Chem. Phys. 81, 335 (1983).

${ }^{50}$ J. Noolandi, K.M. Hong and R.A. Street, Solid State Commun. 34, 45 (1980).

${ }^{51}$ A. Mozumder, J. Chem. Phys. 48, 1659 (1968).

${ }^{52}$ P.G. de Gennes, P. Lafore, and J.P. Millot, J. Phys. Chem. Solids 11, 105 (1966).

${ }^{53}$ P.G. de Gennes, P. Lafore, and J.P. Millot, J. Phys. Radium 20, 624 (1959).

${ }^{54}$ J.W. Essam, in Phase Transitions and Critical Phenomena, edited by C. Domb and M.S. Green (Academic Press, London, 1972), Vol. 2, chap. 6, p. 197.

${ }^{55}$ J.A. Van Vechten and T.K. Bergstresser, Phys. Rev. B 1, 3351 (1970).

${ }^{56}$ D. Richardson, J. Phys. C 5, L27,L127 (1972).

${ }^{57}$ A. Baldereshi and K. Mashke, Solid State Commun. 16, 99 (1975).

${ }^{58}$ R.J. Elliott, J.A. Krumhansl, and P.L. Leath, Rev. Mod. Phys. 46, 465 (1974).

${ }^{59}$ B. Velicky, S. Kikpatrick, and H. Erenreich, Phys. Rev. 175, 747 (1968).

${ }^{60}$ K.C. Hass, B. Velicky, and H. Ehrenreich, Phys. Rev. B 29, 3697 (1984).

${ }^{61}$ S. Krishnamurthy, M.A. Berding, A. Sher, and A.-B. Chen, Phys. Rev. B 37, 4254 (1988).

${ }^{62}$ A.-B. Chen, Semiconductor Alloys: Physics and Materials Engineering, edited by A. Sher and A.-B. Chen (Plenum Press, New York, 1995).

${ }^{63}$ J.E. Bernard and A. Zunger, Phys. Rev. B 36, 3199 (1991).

${ }^{64}$ S.-H. Wei, L.G. Ferreira, J.E. Bernard, and A. Zunger, Phys. Rev. B 42, 9622 (1990)

${ }^{65}$ S.-H. Wei and A. Zunger, Phys. Rev. B 43, 1662 (1991).

${ }^{66}$ S.-H. Wei and A. Zunger, Phys. Rev. B 39, 3279 (1991).

67 B. Segal and D.T.F. Marple, in Physics and Chemistry of II-VI Semiconducting Compounds, edited by M. Aven and J.S. Prener (North-Holland, Amsterdam, 1967), p. 319.

${ }^{68}$ G.F. Koster and J.C. Slater. Phys. Rev. 95, 1167 (1954).

${ }^{69}$ I.M. Lifshitz, Nuovo Cimento 3, Suppl. Series X, 716 (1956).

${ }^{70}$ M. Lannoo and P. Lenglart, J. Phys. Chem. Solids 30, 2409 (1969).

${ }^{71}$ J. Bernholc and Sokratas Pantelides, Phys. Rev. B 18, 1780 (1978).

${ }^{72}$ O. Goede, W. Heimbrodt, and R. Müller, Phys. Status Solidi B 105, 543 (1981).

${ }^{73}$ A.A. Maradudin, Rep. Prog. Phys. XXVIII, 331 (1965).

${ }^{74}$ In general, we have to consider two sets of eigenvectors of matrix
Eq. (24), details can be found in: Nguen Xuan Xinh, A.A. Maradudin, and R.A. Coldwell-Horsfall, J. Phys. (France) 26, 717 (1965).

${ }^{75}$ A.I. Baz', Ya. B. Zel'dovich, and A.M. Perelomov, Scattering, Reactions, and Decay in Nonrelativistic Quantum Mechanics (Nauka, Moskow, 1971).

${ }^{76}$ R.G. Newton, Scattering Theory of Waves and Particles (McGraw-Hill, New York, 1966).

${ }^{77}$ I.Ch. Akopyan, D.M. Gromov, A.A. Klochikhin, B.V. Novikov, and S.G. Ogloblin, Phys. Status Solidi B 148, 185 (1989).

${ }^{78}$ B.I. Halperin and M. Lax, Phys. Rev. 148, 722 (1966).

${ }^{79}$ B.I. Halperin and M. Lax, Phys. Rev. 153, 802 (1966).

${ }^{80}$ P.W. Anderson, Phys. Rev. 109, 1492 (1958).

${ }^{81}$ D.J. Thouless, Phys. Rep. 13C, 95 (1974).

${ }^{82}$ Y. Nagoka, Progr. Theor. Phys. Suppl. 84, 1 (1985).

${ }^{83}$ Anderson Localization, edited by T. Ando and H. Fukuyama (Springer, Berlin, 1988).

${ }^{84}$ W.Y. Ching and D.L. Huber, Phys. Rev. B 25, 1096 (1982).

${ }^{85}$ Y. Meir, A. Aharony, and A.B. Harris, Europhys. Lett. 10(3), 275 (1989).

${ }^{86}$ I. Chang, Zvi Lev, A.B. Harris, J. Adler, and A. Aharony, Phys. Rev. Lett. 74, 2094 (1995).

${ }^{87}$ A.A. Maradudin, Solid State Phys. 18, 273 (1966); 19, 1 (1966).

${ }^{88}$ M.H. Pryce, in Phonons, edited by R.W.H. Stevenson (Plenum, New York, 1966).

${ }^{89}$ A.I. Ansel'm and Yu. A. Firsov, Zh. Exp. Teor. Fiz. 28, 151 (1955) [Sov. Phys. JETP 1, 139 (1955)].

${ }^{90}$ Physics and Chemistry of II-VI Compounds, edited by M. Aven and J.S. Prener (North-Holland, Amsterdam, 1967).

${ }^{91}$ P. Grassberger and I. Procaccia, J. Chem. Phys. 77, 6281 (1982).

${ }^{92}$ S. Alexander and R. Orbach, Phys. Lett. 43, L-625 (1982).

${ }^{93}$ T. Nakayama, K. Yakubo, and R. Orbach, Rev. Mod. Phys. 66, 381 (1994).

${ }^{94}$ It is possible to expect that the scale of the conduction band tailing is considerably less than that of the valence band in anion-substituted materials. This difference in the scales of the tails makes the tail of the conduction band rather nonobservable in the absorption or in the steady state luminescence spectra. The luminescence spectra in the long-time limit coincide practically with the exciton-phonon bands at steady-state conditions. Therefore, the process of the exciton formation from separated pairs has to occur before the radiative recombination of the particles. The rate of this process depends on the nature of the electron states and we can expect that, as the consequence, the electron tailing influences the process of the luminescence kinetics. 